\title{
Diagnostic accuracy of AS-OCT vs gonioscopy for detecting angle closure: a systematic review and meta-analysis
}

\author{
Thomas Desmond $^{1,2}$ (D) $\cdot$ Vincent Tran $^{2} \cdot$ Monish Maharaj ${ }^{3,4} \cdot$ Nicole Carnt $^{1,2,5,6} \cdot$ Andrew White $^{1,2,5}$
}

Received: 12 January 2021 / Revised: 13 May 2021 / Accepted: 3 June 2021 / Published online: 5 July 2021

(c) The Author(s), under exclusive licence to Springer-Verlag GmbH Germany, part of Springer Nature 2021

\begin{abstract}
Purpose This study aims to review the literature that compares the accuracy of Anterior Segment-Optical Coherence Tomography (AS-OCT) against gonioscopy in detecting eyes with angle closure. It is currently unclear how AS-OCT fits into clinical practice for detecting angle closure. This is a systematic review and meta-analysis.

Methods A literature search was performed on Medline, Embase, Scopus and the Cochrane Central Register of Controlled Trials to identify studies that investigated the diagnostic accuracy of AS-OCT in detecting eyes with angle closure as diagnosed by gonioscopy. Eligible studies included in the analysis met stringent inclusion criteria determining the sensitivity and specificity of AS-OCT.

Results The initial search identified 727 studies, of which 23 were included in the final analysis. We found substantial variation in the parameters being studied and methodologies. The sensitivity of AS-OCT ranged from 46 to $100 \%$ (median $87 \%$ ). Twenty-one studies identified parameters that showed sensitivity above $80 \%$. The specificity ranged from 55.3 to $100 \%$ (median $84 \%$ ).

Conclusion AS-OCT demonstrates good sensitivity for detecting angle closure. It may provide an avenue to address high rates of undiagnosed angle closure, such as found in developing Asian countries. However, AS-OCT is not yet able to replace gonioscopy. Clinicians should consider whether the diagnostic accuracy of AS-OCT is acceptable for their specific clinical use before adopting it. More studies are needed to determine the utility of AS-OCT, including longitudinal studies to determine the significance of eyes classified to have closed angles by AS-OCT but open on gonioscopy.
\end{abstract}

Keywords Anterior segment optical coherence tomography $\cdot$ OCT $\cdot$ Glaucoma $\cdot$ Narrow angle $\cdot$ Gonioscopy $\cdot$ Screening

Thomas Desmond

thomas@thomasdesmond.net

1 Sydney Medical School, University of Sydney, Sydney, Australia

2 School of Optometry and Vision Science, University of New South Wales, Sydney, Australia

3 Faculty of Medicine, University of New South Wales, Sydney, Australia

4 Department of Neurosurgery, Waikato Hospital, Hamilton, New Zealand

5 Westmead Institute for Medical Research, Sydney, Australia

6 Institute of Ophthalmology, University College London, London, UK 


\section{Key messages}

- It is currently unclear how AS-OCT fits into clinical practice for detecting angle closure.

- AS-OCT is sensitive for detecting angle closure.

- AS-OCT may be a good screening tool for angle closure.

- AS-OCT has a high rate of false positives when measured against gonioscopy.

- AS-OCT is not yet able to replace gonioscopy.

\section{Introduction}

Primary angle closure glaucoma (PACG) affects an estimated 23 million people worldwide, with over $80 \%$ of cases found in Asia [1]. PACG is a more aggressive form of glaucoma, and despite accounting for only around $26 \%$ of glaucoma cases, it causes almost half of the blindness [2]. It confers a three times higher risk of blindness compared to primary open angle glaucoma (POAG) [2-4]. Angle closure disease is significantly underdiagnosed worldwide, particularly in rural or developing areas, causing a significant burden of blindness. In China, over $90 \%$ of primary angle closure cases were undiagnosed in 2010 [5]. PACG is also frequently misdiagnosed, with up to two-thirds of cases misdiagnosed as POAG in one Indian study [6].

Identifying eyes with angle closure, including primary angle closure suspects (PACS), is critical for several reasons. Firstly, PACG is a more aggressive disease than POAG and there is a greater risk of vision loss if left untreated [2]. Almost $30 \%$ of eyes with angle closure and peripheral anterior synechiae or high pressures will progress to PACG within 5 years [7]. Secondly, the treatment for PACG is different to POAG. Treatment can effectively reduce the intraocular pressure and reduce risk of glaucomatous progression. Finally, it may identify eyes at higher risk of acute angle closure with mydriatic agents [8].

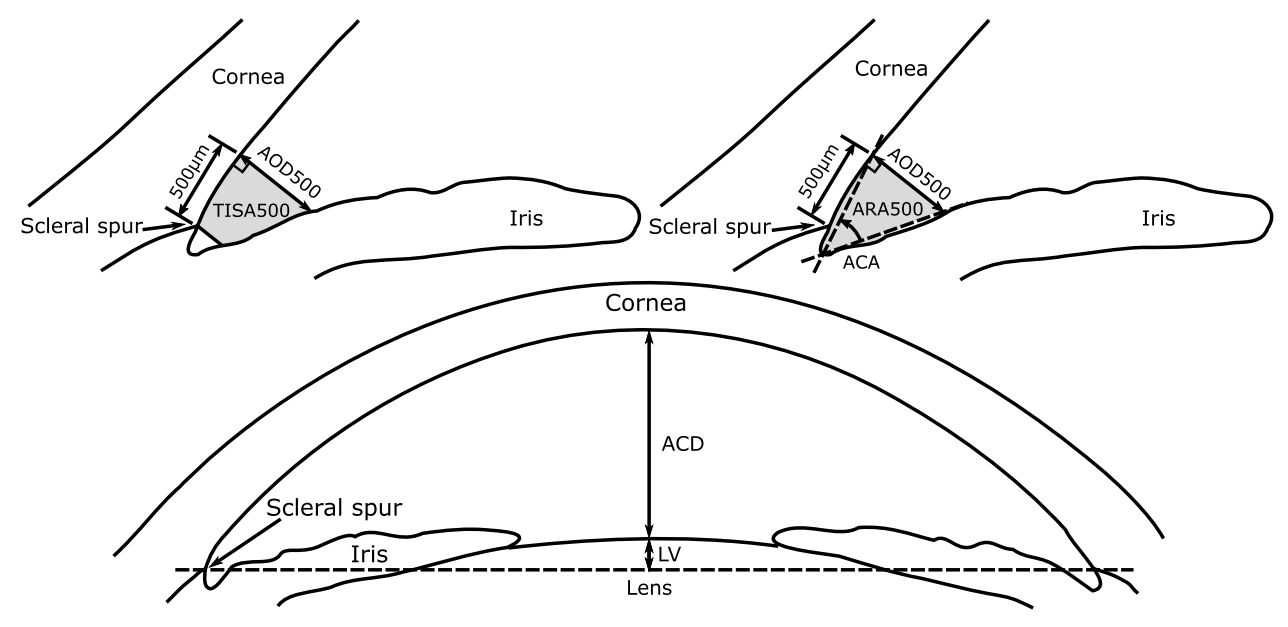

Fig. 1 Angle opening distance at $500 \mu \mathrm{m}$ (AOD500) - the distance between the corneal endothelium and iris along a line drawn perpendicularly from a point on the corneal endothelium 500 microns anterior to the scleral spur. Trabecular-iris space area at $500 \mu \mathrm{m}$ (TISA500) - the area enclosed by the AOD500 line, the anterior iris surface, the corneal endothelium/trabecular meshwork and a line drawn perpendicular from the scleral spur. Angle recess area at $500 \mu \mathrm{m}$ (ARA500) - the area enclosed by the AOD500 line, anterior iris surface and the corneal endothelium/trabecular meshwork/ciliary body. Anterior chamber angle (ACA) - this may be formally defined using the trabecular-iris angle at $500 \mu \mathrm{m}$ (TIA500). The angle subtended by the AOD500 line from the apex of the iris recess. Lens vault (LV) - the perpendicular distance between the anterior apex of the lens and the line that joins the two opposite scleral spurs. Anterior chamber depth (ACD) - the distance between the corneal endothelium and anterior surface of the lens along the central axis. Anterior chamber volume (ACV) - the volume of the anterior chamber. Iridotrabecular contact index (ITC index) - a parameter that reflects the circumferential extent of anterior angle with iridocorneal touch. Iridotrabecular contact length (ITC length) - the distance between the scleral spur and the anterior extent of iridocorneal touch. Iridotrabecular contact area (ITC area) - the total area of ITC in the eye, determined by ITC length around the circumference of the eye. Trabecular-iris circumference volume at $500 \mu \mathrm{m}$ (TICV500)- the total volume of trabecular-iris space in the eye, determined by TISA500 around the circumference of the eye 
Detection of angle closure relies on careful assessment of the anterior chamber angles. The gold standard method is gonioscopy [9]. This can be performed with basic equipment and allows 360-degree visualisation of the anterior chamber angle. However, it has several downsides including that it requires a significant amount of skill from the practitioner, compliance from the patients, contact with the eye and only fair repeatability [10]. The skill required is a significant barrier to effective detection of angle closure. Very few clinicians can perform gonioscopy well, contributing to the high rate of undiagnosed angle closure disease. In a USA-based study, optometrists were $46 \%$ less likely to diagnose angle closure disease than ophthalmologists [11]. Poorer countries are even less likely to have skilled clinicians and the equipment needed for assessment by gonioscopy. For these reasons, gonioscopy is not suitable for large scale screening. There is a need for better screening tools to improve detection rates of angle closure, especially in rural areas or developing countries [12].

Anterior segment optical coherence tomography (ASOCT) is a computerised imaging technology that provides optical cross-sectional images of ocular structures. It has become an invaluable tool for assessing the anterior segment as it provides high-resolution visualisation of the cornea and anterior chamber as well as objective measures of anterior eye parameters (Fig. 1).

Although AS-OCT can provide excellent data on the structure of the anterior chamber angle, it remains unclear how it fits into clinical practice for detecting angle closure. The largest review by the American Academy of Ophthalmology was conducted on studies up from 2005 to 2011 and concluded that AS-OCT could provide useful supplemental information when used alongside gonioscopy [13]. A review by Porporato et al. (2018) found that AS-OCT had good sensitivity and diagnostic accuracy but did not include several studies of lower level of evidence [14]. Both reviews did not focus on collating sensitivity and specificity data to compare AS-OCT against gonioscopy. A review by Chansangpetch et al. (2018) did collate sensitivity and specificity data but did not conduct the review systematically [15]. A Cochrane review by Jindal et al. (2020) assessed non-contact tests for angle closure but did not compare against gonioscopy as a reference standard [16].

This study aimed to systematically review the literature investigating the diagnostic accuracy AS-OCT in the detection of angle closure, thus determining the utility of AS-OCT in clinical practice. This systematic review was conducted with a focus on reviewing the sensitivity and
Fig. 2 Study selection flowchart following the PRISMA guidelines

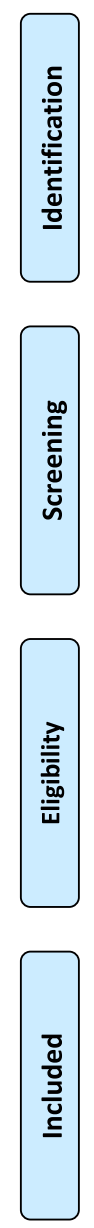


specificity of parameters that can be measured with ASOCT when compared against gonioscopy as the reference standard (Fig. 2).

\section{Methods}

This paper was written in accordance with the 1964 Declaration of Helsinki and its later amendments. The University of Sydney ethics committee waived the need for ethics approval due to this paper being a literature review. Data was gathered by electronic searches of MEDLINE, EMBASE, Scopus and the Cochrane Central Register of Controlled Trials (CENTRAL). Search terms included, 'gonio*', 'optical coherence tomography', 'angle closure', 'narrow angle', 'diagnos*', 'identif*', 'screen*' and 'detect*'. The literature search was performed in April 2020. The reference lists of included studies were reviewed to search for any papers that may have been missed during this search. Only papers written in English were included in this review. Only studies performed on humans were included. Abstracts, case studies and expert opinions were excluded from this review.

Selection criteria included any trial that investigated the diagnostic accuracy of AS-OCT in detecting eyes with angle closure as diagnosed by gonioscopy. Only papers that provided enough data to determine the sensitivity and specificity of AS-OCT were included. Only papers that assessed the ability to detect an eye with angle closure (as opposed to a quadrant or individual with angle closure) were included.

After the literature search was performed, study selection, extraction of data and assessment of risk of bias were independently performed by two key investigators (TD and VT). For any papers with missing data, the corresponding author would be contacted to find the missing data. If no reply was received 2 weeks after initial contact, then the study would be excluded. An assessment of each studies risk of bias was performed using the QUADAS-2 tool [17]. We modified the QUADAS-2 tool to also consider whether each study analysed only one eye per participant. Any disagreement was discussed amongst the two investigators to resolve the discrepancy.

Data was extracted by two key investigators (TD and VT) for each index parameter in each study. We extracted the number true positives, false positives, false negatives and true negatives to construct $2 \times 2$ contingency tables. This approach allows calculation of sensitivity and specificity as well as positive and negative predictive values (PPV and NPV). We only reported PPV and NPV values for studies that did not use a case-control design. When data was given for both training and validation sets, only results from the validation set were extracted as we deemed them to be statistically more rigorous. When data were included in more than one publication, only data from the most recent publication were extracted for analysis. To present the most relevant data, analysis of studies with a longitudinal design was performed and reported separately. This was necessary because they had different approaches and aims when investigating the clinical question.

Data was entered into Revman 5.4 [18] to create the forest plots presented in this review. We performed a meta-analysis of the sensitivity and specificity data when there were data from 3 or more studies available for an index parameter. This was performed using a hierarchical summary receiver operator curve (ROC) model for each index parameter [19]. Results were calculated using the MetaDAS macro [20] in SAS for Windows, version 9.4 [21] and presented as an estimated summary ROC curve. The Cochrane Handbook outlines how estimation of a summary ROC curve is the most appropriate form of meta-analysis given the studies included in our review [19]. Unfortunately, it was not appropriate to calculate summary sensitivity and specificity points for any index parameters because there were few studies available or there was large heterogeneity in gonioscopy criteria and/or positivity threshold. If we were to calculate summary sensitivity and specificity values, they would represent diagnostic accuracy at an indeterminate average positivity threshold and be unusable in clinical practice [19].

Some studies provided data on more than one index parameter or at more than one positivity threshold. Our analysis only included a single data set per index parameter per study. When there was more than one data set available for an index parameter in a study, such as when multiple positivity thresholds were tested, the single data set with the highest Youden's $J$ statistic was selected for analysis [22].

Comparison between index parameters was made directly by analysing studies that assessed more than one index parameter. Takwoingi et al. (2013) [23] showed that this approach is more reliable than indirect comparisons. We extended this to allow for direct comparison between studies by the same researchers that used the exact same patients and study design and only differed in the index parameter being studied. It was not possible to perform a formal statistical comparison between index parameters due to the small number of studies for each index parameter.

It was not possible to perform a quantitative heterogeneity analysis for any index parameter due to a small number of studies. Instead, we have presented a narrative description of the contributors to heterogeneity, aided by graphical presentation on the relevant summary ROC plot.

\section{Results}

The initial search gave 727 papers across the 4 databases. After removing duplicates, there were 373 papers to be reviewed. These papers were screened based on their title and abstract and after removing unsuitable studies, there 
were 76 papers remaining for a full text screen. Twentyfive papers were found to be suitable for inclusion after full appraisal. Two further studies were excluded. One for missing data and the authors not contactable [24], and the other because there was a more in-depth reanalysis of the same data in another later study $[25,26]$.

Seven studies were performed by the same research group and used a common set of patients, labelled as Population A [26-32]. Another two studies used another common set of patients, labelled as Population B [33, 34]. These studies tested different index parameters; therefore, it was not necessary to entirely exclude any of the studies. Occasionally, some data were presented in more than one study. Accordingly, subsets of data from two studies were excluded to avoid double reporting of the data [27, 33].

A total of 5663 patients were included across all studies, excluding the duplicate patients that appeared in more than one study. Eighteen studies $(78 \%)$ were based out of Asia, 3 out of the USA (13\%), and 2 out of the UK (9\%). Ten studies $(43 \%)$ were conducted in a community setting, $12(52 \%)$ were conducted in a secondary care setting, and in 1 study, it was unclear (4\%). Two studies were longitudinal in nature and investigated the accuracy of ASOCT in predicting eyes that would develop angle closure 4 years later [32]. Results from 7 different OCT devices were analysed between the included studies. Characteristics of each study are outlined in Table 1.

Gonioscopy criteria for diagnosing angle closure varied between studies. Non-visibility of posterior trabecular meshwork (PTM) was used as gonioscopy criteria for diagnosing angle closure in most studies. Non-visibility of $\geq 1,2,3$ and 4 quadrants of PTM was used as a threshold in 5, 13, 6 and 1 studies, respectively. Six studies did not directly assess for visibility of PTM and used the Spaeth grading system or a modified Shafer grading system instead $[37,40]$. One study analysed only three quadrants in each eye to determine angle closure by gonioscopy [47].

The risk of bias was found to be variable amongst the studies and summarised in Figs. 3 and 4. We identified two areas of study design that were frequently performed in a way that are likely to introduce bias. Eight studies did not avoid a case-control study design which falls under the 'Patient Selection domain'. Nine studies did not pre-specify a threshold for diagnosis of angle closure which falls

Table 1 Study characteristics

\begin{tabular}{|c|c|c|c|c|c|c|}
\hline First Author & Date & No. of eyes & Location & Setting & $\begin{array}{l}\text { Angle closure prevalence } \\
\text { by gonioscopy }(\%)\end{array}$ & Type of OCT \\
\hline Porporato [34] & 2019 & $1865^{\mathrm{B}}$ & Singapore & Community & 7.5 & CASIA SS-1000, Tomey \\
\hline $\mathrm{Li}[35]$ & 2019 & $252^{\mathrm{a}}$ & China & Secondary & N/A & CASIA SS-1000, Tomey \\
\hline Porporato [33] & 2018 & $1857^{\mathrm{B}}$ & Singapore & Community & 5.17 & CASIA SS-1000, Tomey \\
\hline Nongpiur [32] & 2017 & $342^{\mathrm{A}}$ & Singapore & Community & N/A & Visante, Carl-Zeiss \\
\hline Tun [36] & 2017 & 202 & Singapore & Secondary & 24.8 & CIRRUS 5000, Carl-Zeiss \\
\hline Melese [37] & 2016 & $189^{\mathrm{b}}$ & USA & Secondary & N/A & CASIA SS-1000, Tomey \\
\hline Baskaran [31] & 2015 & $342^{\mathrm{A}}$ & Singapore & Community & N/A & Visante, Carl-Zeiss \\
\hline Dabasia [38] & 2015 & 78 & UK & Secondary & N/A & Visante, Carl-Zeiss \\
\hline Campbell [39] & 2015 & 78 & UK & Community & 15 & 3D OCT-2000, Topcon \\
\hline Qin [40] & 2013 & 65 & USA & Secondary & N/A & RTVue, Optovue \\
\hline Nongpiur [26] & 2013 & $1368^{\mathrm{A}}$ & Singapore & Community & 21.6 & Visante, Carl-Zeiss \\
\hline Baskaran [41] & 2013 & 140 & Singapore & Secondary & 22.9 & CASIA SS-1000, Tomey \\
\hline Baskaran [42] & 2012 & 97 & Singapore & Secondary & 39.8 & Visante, Carl-Zeiss \\
\hline Tan [30] & 2012 & $1465^{\mathrm{A}}$ & Singapore & Community & 21.5 & Visante, Carl-Zeiss \\
\hline Grewal [43] & 2011 & 265 & India & Secondary & 10.6 & RTVue 100, Optovue \\
\hline Chang [27] & 2011 & $2047^{\mathrm{A}}$ & Singapore & Community & 19.3 & Visante, Carl-Zeiss \\
\hline Narayanaswamy [29] & 2010 & $1465^{\mathrm{A}}$ & Singapore & Community & 21.5 & Visante, Carl-Zeiss \\
\hline Khor [28] & 2010 & $1853^{\mathrm{A}}$ & Singapore & Community & $16.4-28.2$ & Visante, Carl-Zeiss \\
\hline Hong [44] & 2009 & 73 & Korea & Unclear & N/A & SL-OCT, Heidelberg \\
\hline Sakata [45] & 2009 & 83 & Singapore & Secondary & 36.1 & Visante and SL-OCT \\
\hline Wong [46] & 2009 & 153 & Singapore & Secondary & $30.1-33.3$ & Visante, Carl-Zeiss \\
\hline Nolan [47] & 2006 & 342 & Singapore & Secondary & 44.4 & Prototype OCT, Carl-Zeiss \\
\hline Radhakrishnan [48] & 2005 & 31 & USA & Secondary & N/A & Prototype OCT, Carl-Zeiss \\
\hline
\end{tabular}

${ }^{\text {a }}$ Seventy-five eyes in validation set. ${ }^{\text {b}}$ Sixty-nine eyes in validation set. ${ }^{\text {A }}$ Shared population A. ${ }^{\mathbf{B}}$ Shared population B 


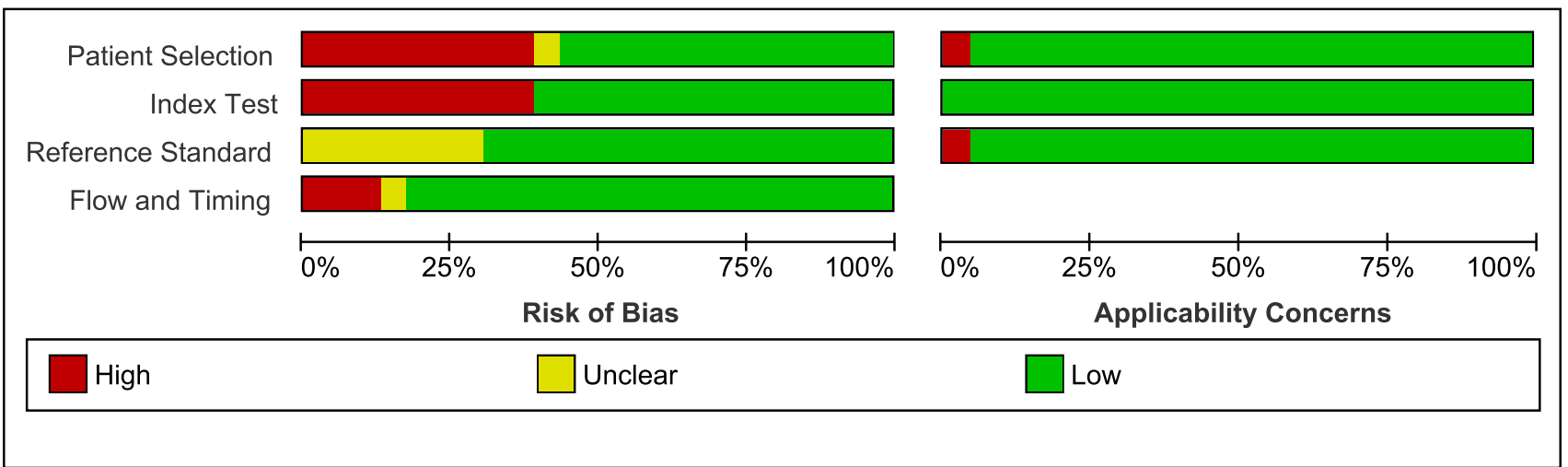

Fig. 3 Risk of bias and applicability concerns graph

under the 'Index Test' domain. Instead, they determined an ideal threshold to fit to the data after it had been collected.

Between all 23 studies, the sensitivity ranged from 46 to $100 \%$ (median $87 \%$ ) and the specificity ranged from 55.3 to $100 \%$ (median $84 \%)$. There were $21(91 \%)$ and $17(74 \%)$ studies that identified a parameter that had sensitivity and specificity over $80 \%$, respectively. The four studies that showed the best diagnostic accuracy for AS-OCT all used a case-control study design [37,35-48]. It is likely that this artificially improved their results. Summarised data from all 23 studies was collated and presented in Table 2 (available online).

\section{AOD and TISA}

AOD and TISA are two parameters that were often analysed together. Six studies assessing AOD were included in the analysis for a total of 1970 participants (Figs. 5 and 6). Five studies assessing TISA were included in the analysis for a total of 1905 participants (Figs. 7 and 8).

The largest study to analyse AOD and TISA was performed by Narayanaswamy et al. in 2010 [29]. They reported that the most powerful parameters were AOD750 nasally (sensitivity $82.5 \%$, specificity $84.0 \%$ ) and AOD750 temporally (sensitivity $90.2 \%$, specificity $77.4 \%$ ). This study reported the lowest specificity for AOD and was the only study conducted in a community setting.

We considered 4 studies to be at high risk of bias in the patient selection domain for not avoiding a case-control design $[37,40,35,48]$. This includes the 3 studies with the highest reported sensitivity for AOD and TISA. We considered 4 studies to be at high risk of bias in the index test domain because they did not pre-specify a positivity threshold $[29,40,48,43]$. We also considered two studies to be at increased risk of bias for analysing more than one eye per patient $[40,48]$.

\section{Iridotrabecular touch}

Seven studies assessing for the presence of iridotrabecular touch were included in analysis for a total of 2808 participants (Figs. 9 and 10). The best evidence comes from a study by Khor et al. (2010)[28]. They found that it was most effective when assessing for touch in the inferior quadrant, reaching a sensitivity as high as $87.8 \%$. However, specificity was less than $73 \%$. The results for this study sit directly on the summary ROC curve. The risk of bias amongst the studies was generally low. All of the studies avoided a case-control design, apart from one study by Campbell et al. (2015) where it is uncertain [39]. The study by Nolan et al. (2006) was considered to be at an increased risk of bias for including more than one eye per patient [47].

\section{ITC index}

Three studies assessing ITC index were included in the analysis for a total of 2074 patients (Figs. 11 and 12). One study does not appear in the analysis as data from the same patients were also presented in an earlier study $[33,34]$. The data from the later study were essentially identical but used different gonioscopy criteria and had a lower Youden's $J$ statistic.

Porporato et al. (2018) assessed ITC index in a community based study and showed a NPV of over $96 \%$ for all tested thresholds [33, 34]. Baskaran et al.(2013) used a hospital-based population which generally showed similar specificity but higher sensitivity [41]. Melese et al. (2016) showed an improved result over both other studies (sensitivity $93 \%$, specificity $84 \%$ ) [37].

The studies by Porporato et al. (2018) and Baskaran et al.(2013) were both considered to have low risk of bias in all domains. The study by Melese et al. (2016) used a 
Fig. 4 Risk of bias and applicability concerns summary

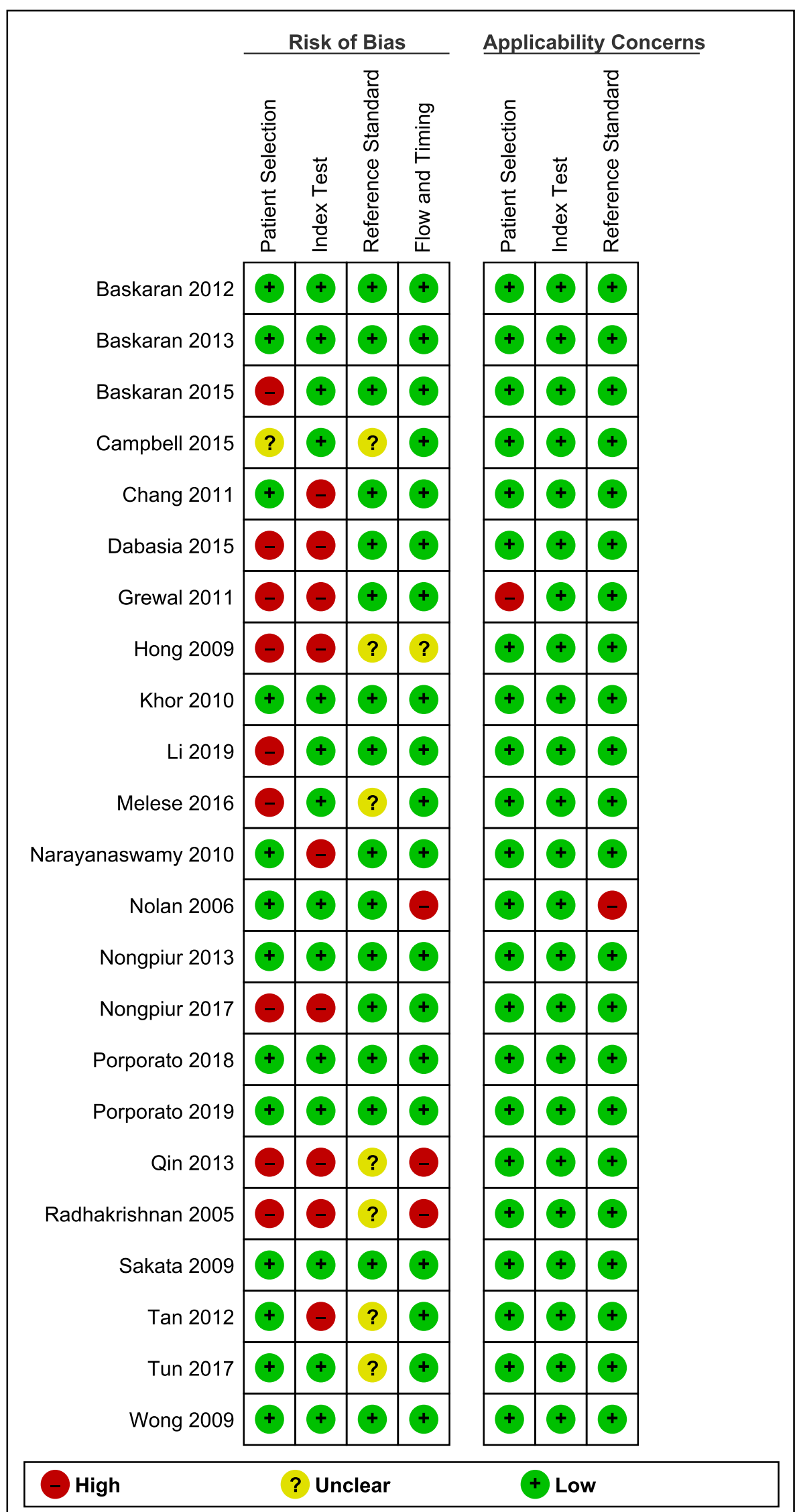




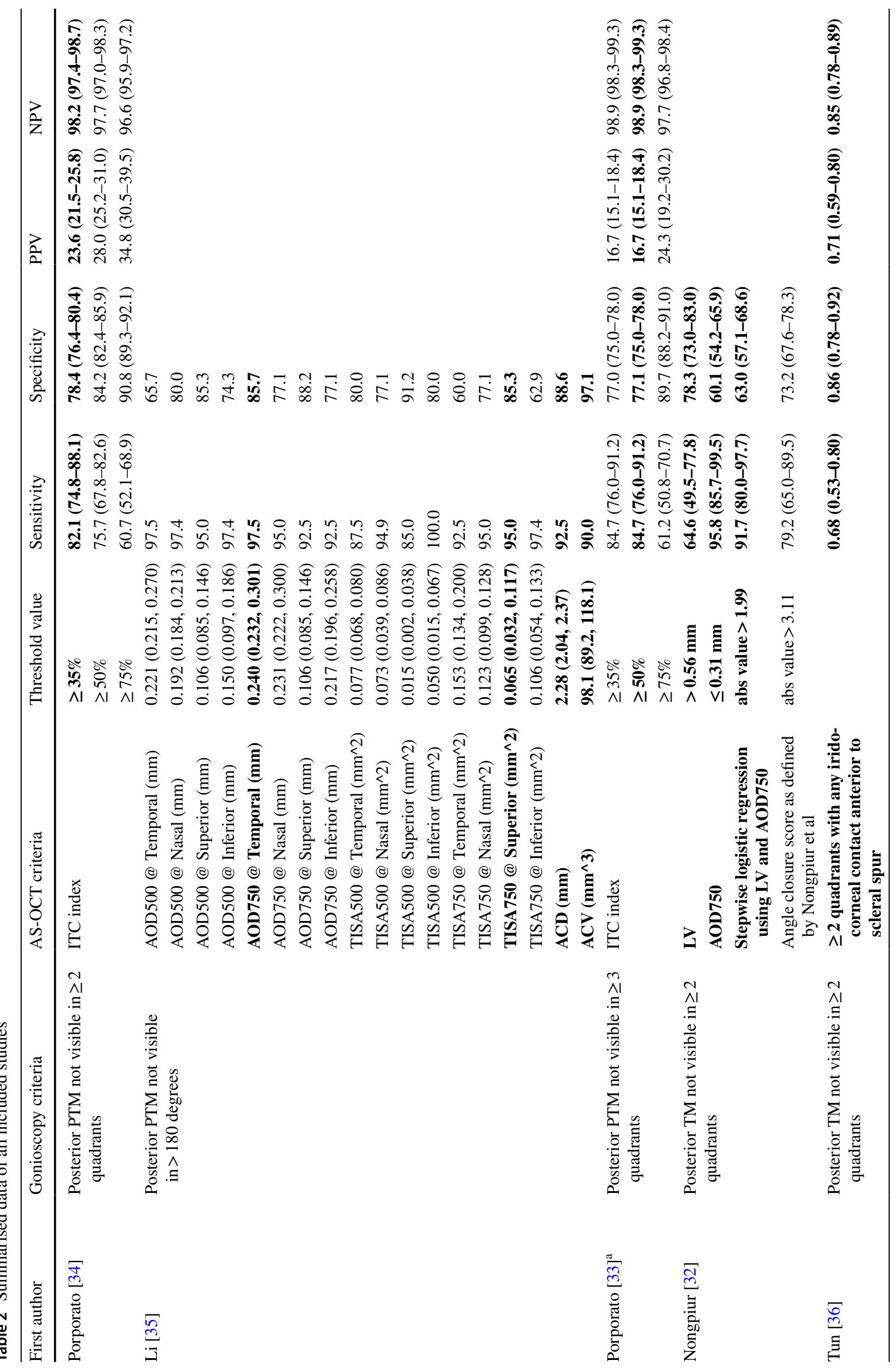




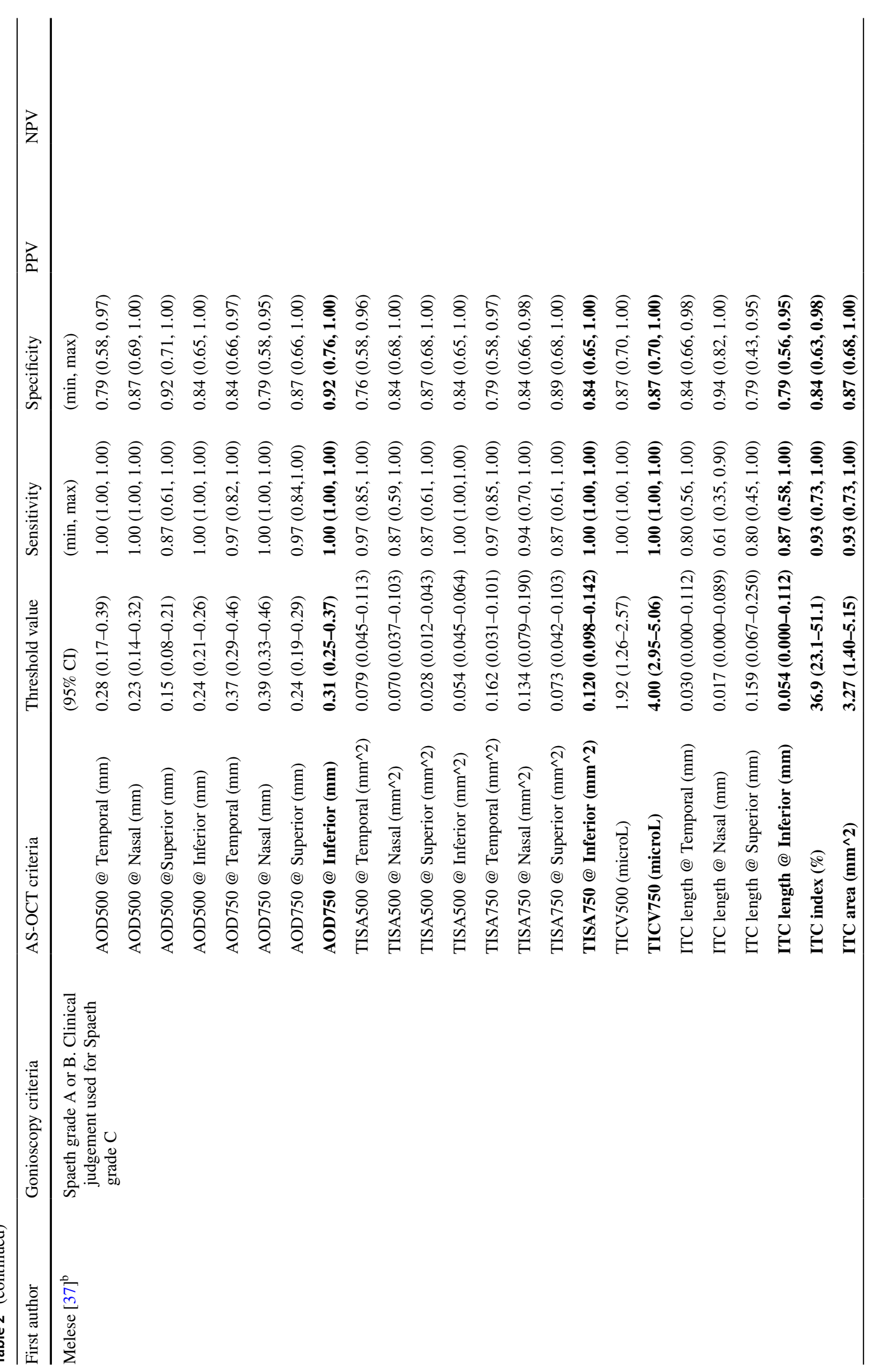




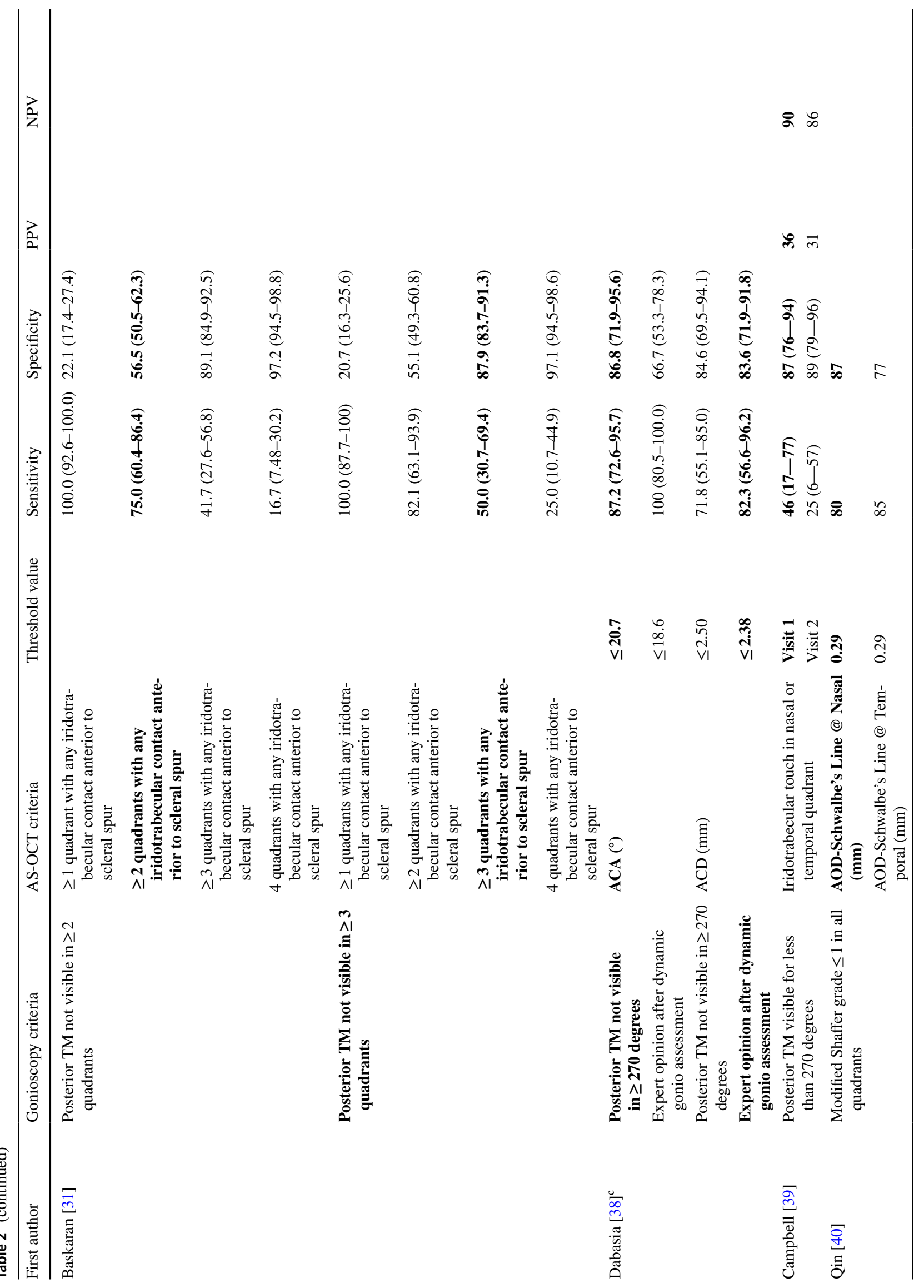




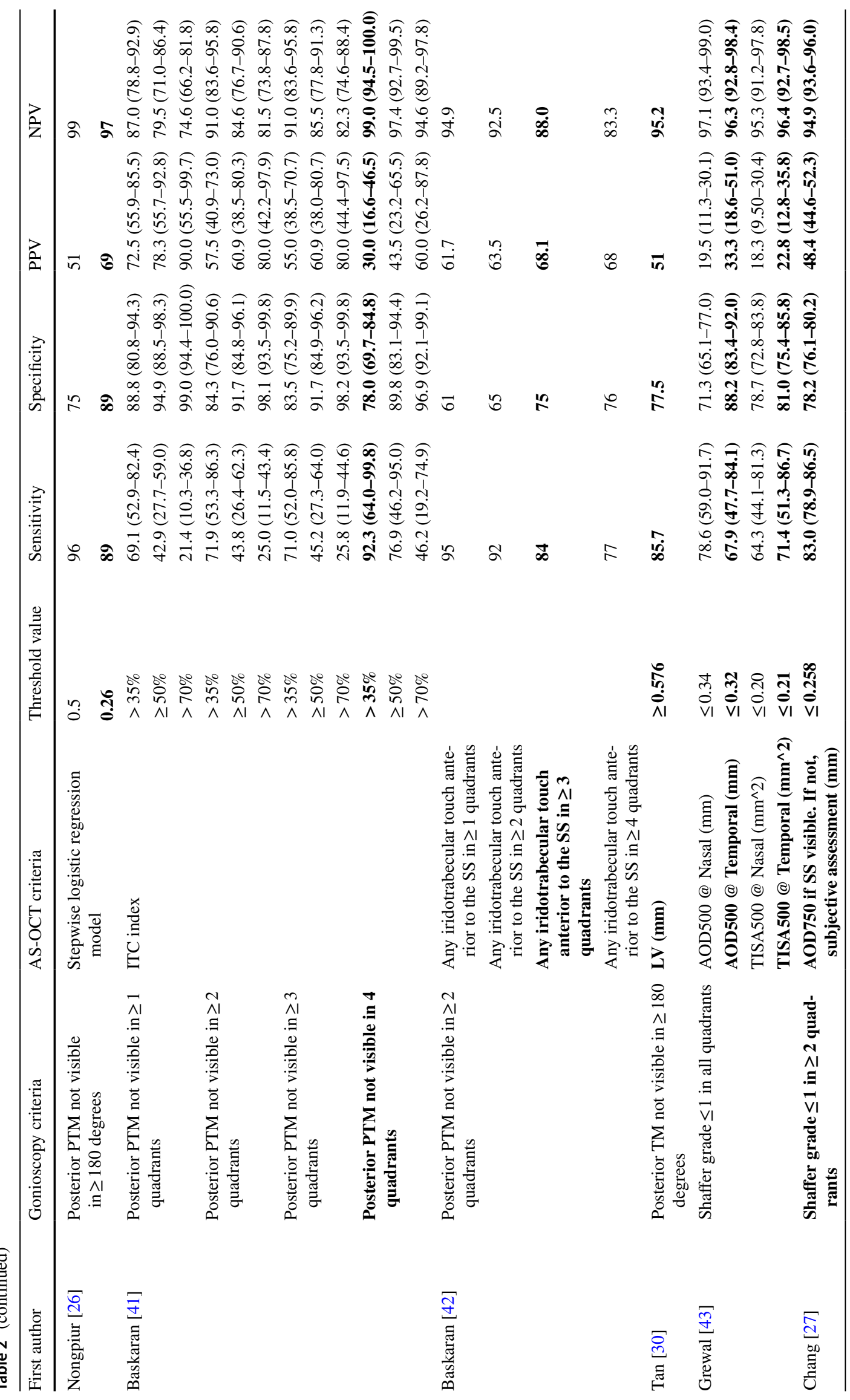




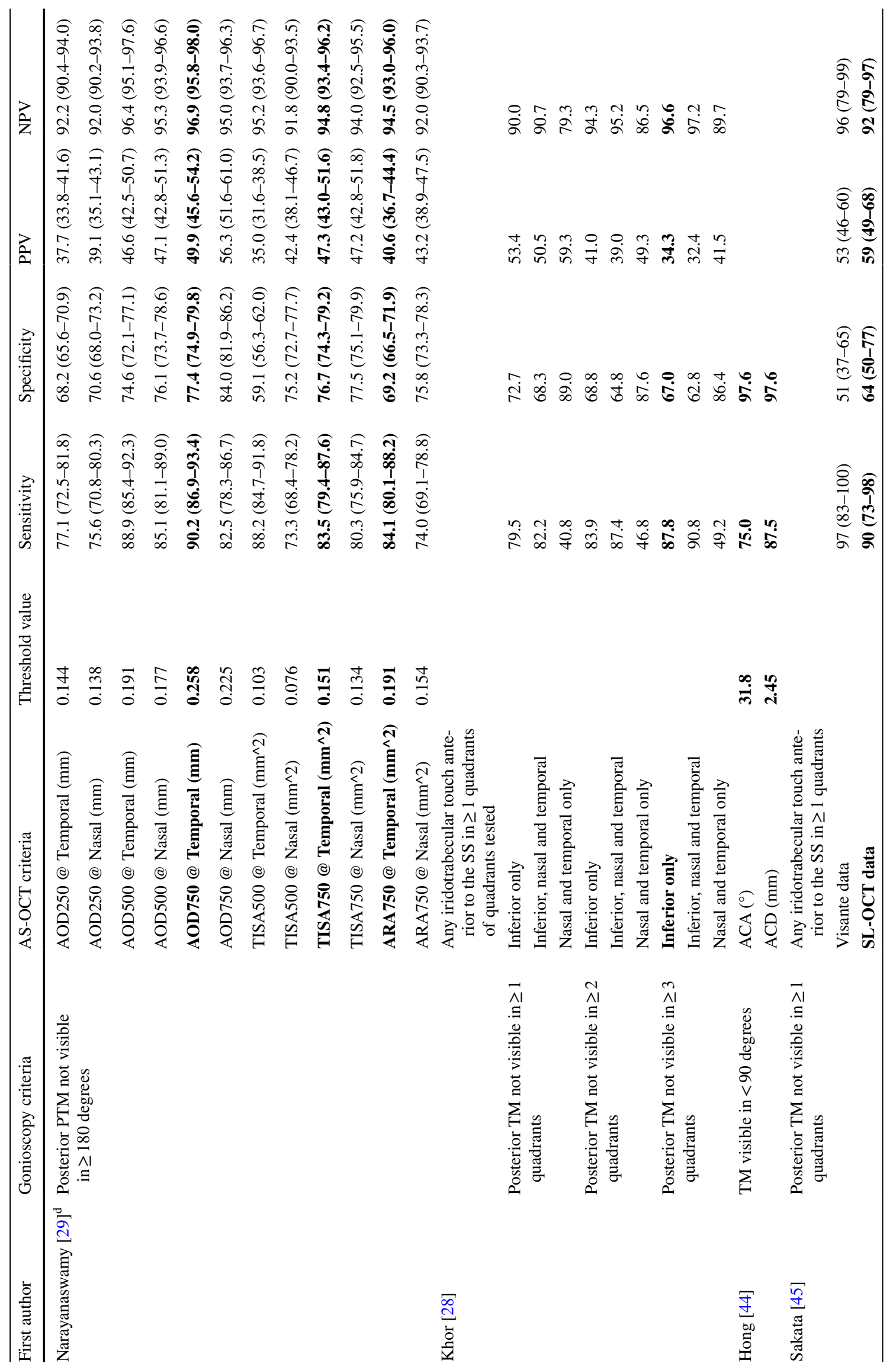




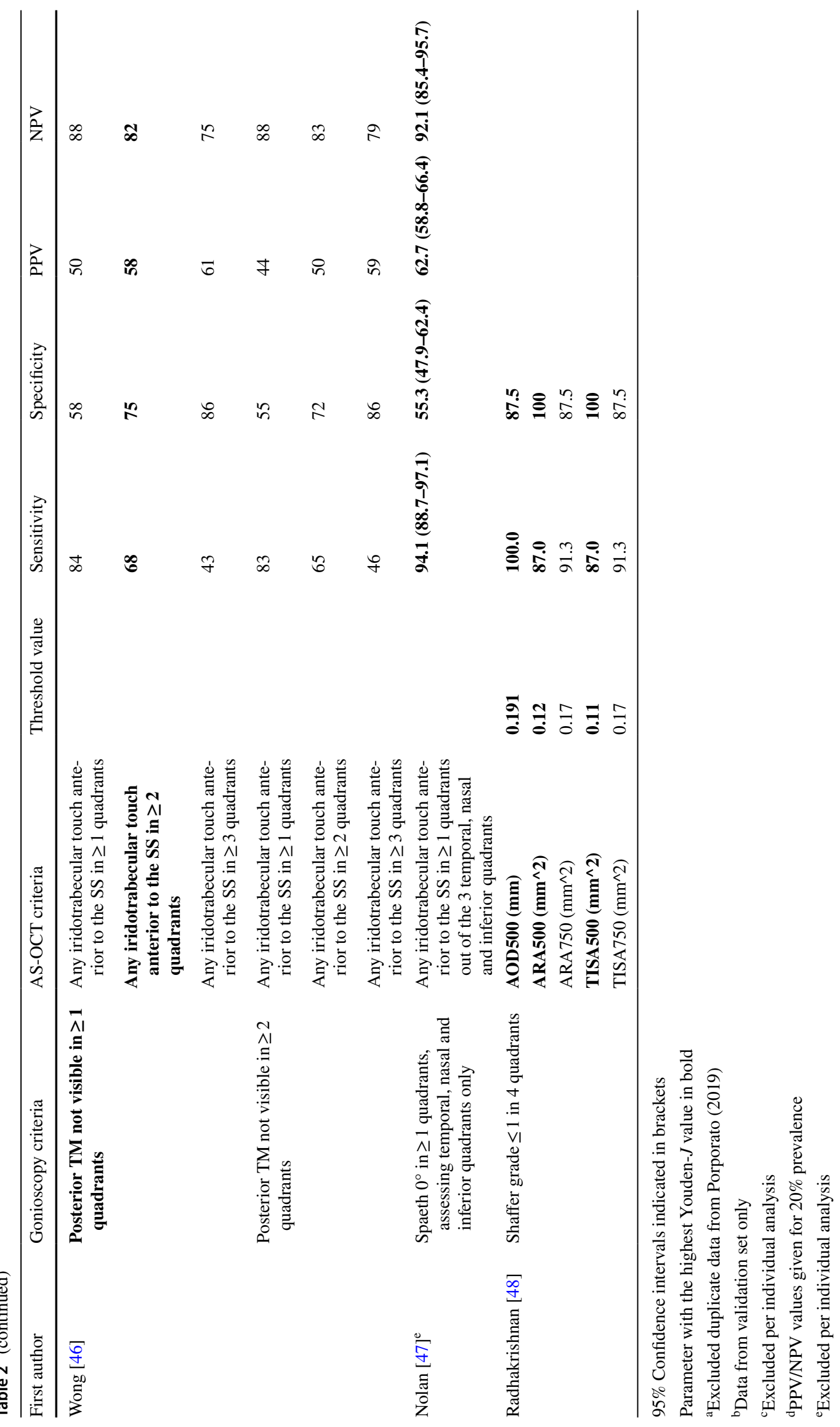




\begin{tabular}{|c|c|c|c|c|c|c|c|c|}
\hline Study & TP & FP & $\mathbf{F N}$ & TN & Sensitivity $(95 \% \mathrm{Cl})$ & Specificity $(95 \% \mathrm{Cl})$ & Sensitivity $(95 \% \mathrm{CI})$ & Specificity $(95 \% \mathrm{Cl})$ \\
\hline Grewal 2011 & 19 & 28 & 9 & 209 & $0.68[0.48,0.84]$ & $0.88[0.83,0.92]$ & & \\
\hline Qin 2013 & 22 & 5 & 6 & 32 & $0.79[0.59,0.92]$ & $0.86[0.71,0.95]$ & & \\
\hline Narayanaswamy 2010 & 284 & 260 & 31 & 890 & $0.90[0.86,0.93]$ & $0.77[0.75,0.80]$ & 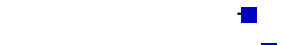 & $\boldsymbol{D}$ \\
\hline Li 2019 & 37 & 5 & 1 & 32 & $0.97[0.86,1.00]$ & $0.86[0.71,0.95]$ & $\rightarrow$ & \\
\hline Radhakrishnan 2005 & 8 & 3 & 0 & 20 & $1.00[0.63,1.00]$ & $0.87[0.66,0.97]$ & & \\
\hline Melese 2016 & 31 & 3 & 0 & 35 & $1.00[0.89,1.00]$ & $0.92[0.79,0.98]$ & 7 & \\
\hline
\end{tabular}

Fig. 5 Forest plot for studies investigating AOD

Fig. 6 Summary ROC plot for studies investigating AOD

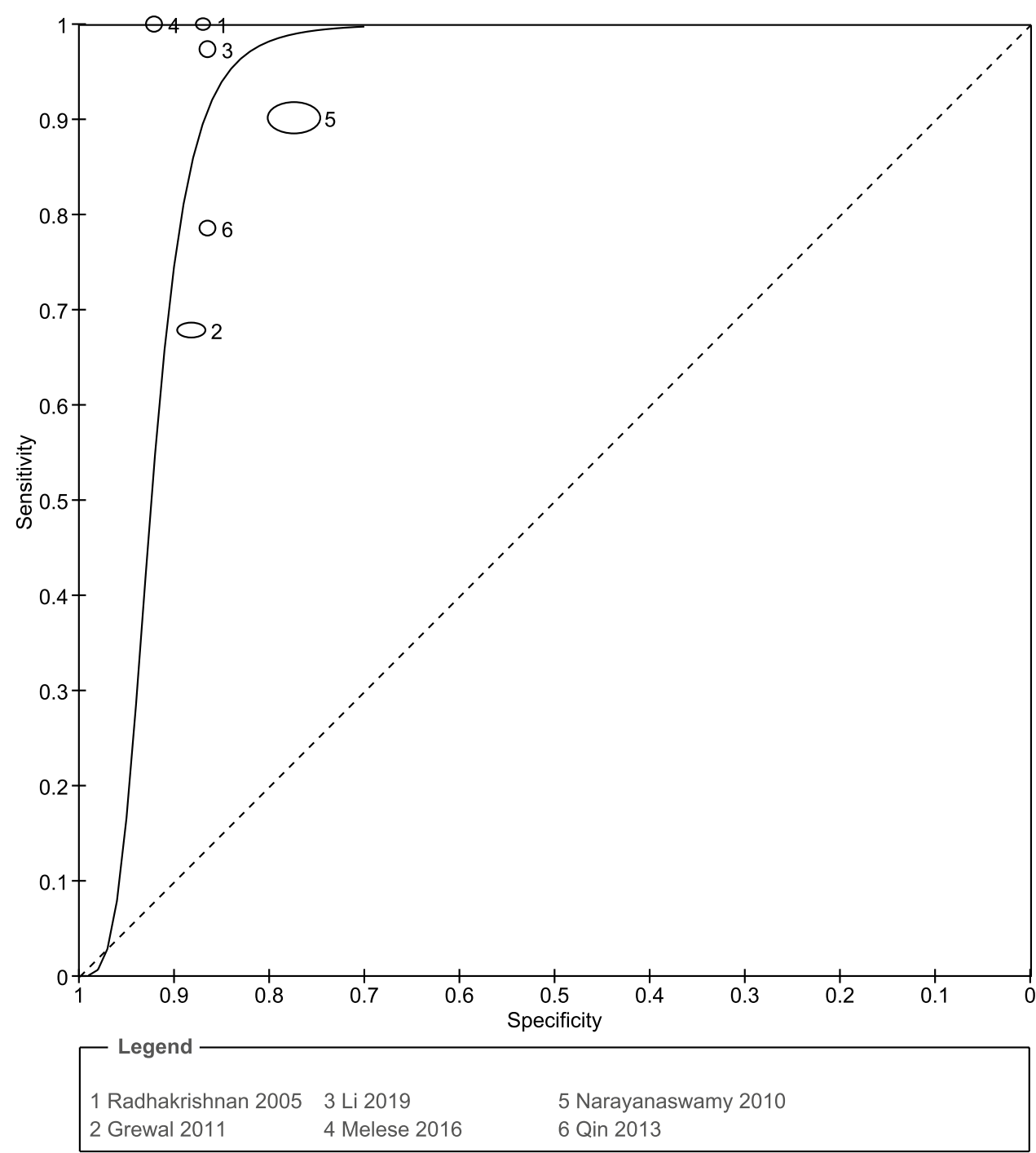

$\begin{array}{lrrrr}\text { Study } & \text { TP } & \text { FP } & \text { FN } & \text { TN } \\ \text { Grewal 2011 } & 20 & 45 & 8 & 192 \\ \text { Narayanaswamy 2010 } & 263 & 268 & 52 & 882 \\ \text { Radhakrishnan 2005 } & 7 & 0 & 1 & 23 \\ \text { Li 2019 } & 36 & 5 & 2 & 32 \\ \text { Melese 2016 } & 31 & 6 & 0 & 32\end{array}$

Study

Narayanaswamy 2010

Melese 2016
Sensitivity $(95 \% \mathrm{Cl})$ Specificity $(95 \% \mathrm{Cl})$ $0.71[0.51,0.87]$ $0.83[0.79,0.87]$

$0.88[0.47,1.00]$

$0.95[0.82,0.99]$

$1.00[0.89,1.00]$
$0.81[0.75,0.86]$

$0.77[0.74,0.79]$

$1.00[0.85,1.00]$

$0.86[0.71,0.95]$

$0.84[0.69,0.94]$
Sensitivity $(95 \% \mathrm{Cl}) \quad$ Specificity $(95 \% \mathrm{CI})$

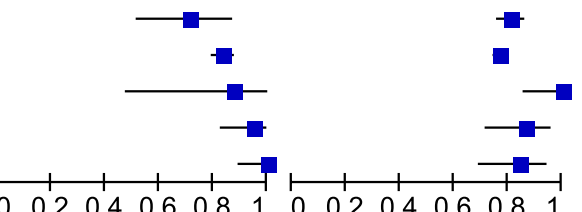

Fig. 7 Forest plot for studies investigating TISA 
Fig. 8 Summary ROC plot for studies investigating TISA

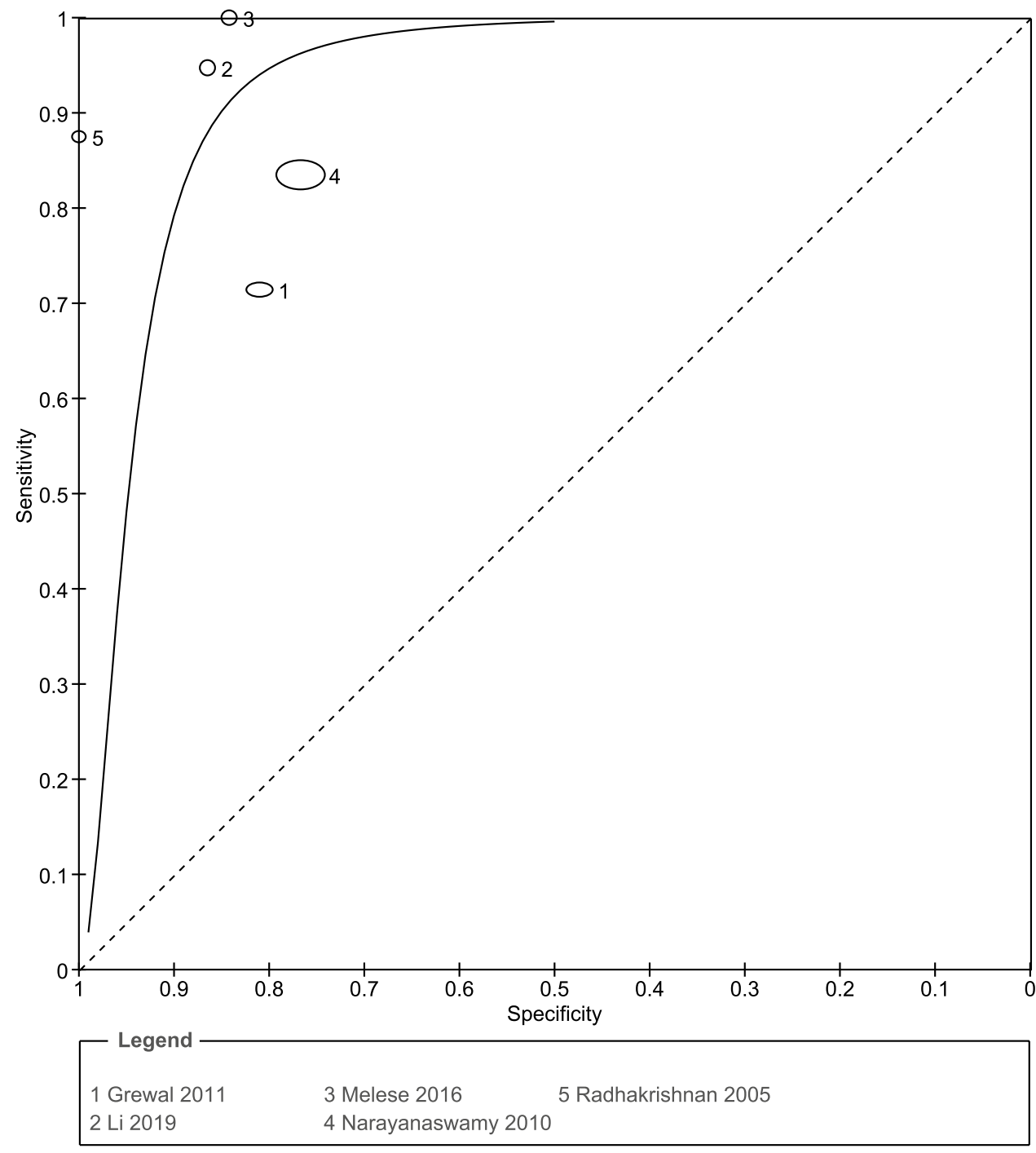

case-control design so we considered it to be at high risk of patient selection bias.

\section{ACD}

Three studies assessing ACD have been included in the analysis for a total of 226 participants (Figs. 13 and 14) [35, 44, 38]. Overall, the evidence investigating ACD is weak due to all 3 studies using a case-control design and small sample sizes. Results showed variable sensitivity (ranging from 71.8 to $92.5 \%$ ) and specificity (ranging from to 83.6 to $97.6 \%$ ).

\section{Remaining index parameters}

The remaining index parameters had few studies to draw data from as shown in the forest plot in Fig. 15. Data has

\begin{tabular}{|c|c|c|c|c|c|c|c|c|}
\hline Study & TP & FP & FN & TN & Sensitivity $(95 \% \mathrm{Cl})$ & Specificity $(95 \% \mathrm{CI})$ & Sensitivity $(95 \% \mathrm{Cl})$ & Specificity $(95 \% \mathrm{Cl})$ \\
\hline Campbell 2015 & 5 & 9 & 6 & 58 & $0.45[0.17,0.77]$ & $0.87[0.76,0.94]$ & & $\rightarrow$ \\
\hline Tun 2017 & 34 & 14 & 16 & 138 & $0.68[0.53,0.80]$ & $0.91[0.85,0.95]$ & & \\
\hline Wong 2009 & 35 & 25 & 16 & 77 & $0.69[0.54,0.81]$ & $0.75[0.66,0.83]$ & & \\
\hline Baskaran 2012 & 32 & 15 & 6 & 44 & $0.84[0.69,0.94]$ & $0.75[0.62,0.85]$ & & $\rightarrow-$ \\
\hline Khor 2010 & 266 & 511 & 37 & 1039 & $0.88[0.84,0.91]$ & $0.67[0.65,0.69]$ & . & घ \\
\hline Sakata 2009 & 27 & 19 & 3 & 34 & $0.90[0.73,0.98]$ & $0.64[0.50,0.77]$ & - & $\longrightarrow$ \\
\hline Nolan 2006 & 143 & 85 & 9 & 105 & $0.94[0.89,0.97]$ & $0.55[0.48,0.62]$ & $\rightarrow$ & - \\
\hline
\end{tabular}

Fig. 9 Forest plot for studies investigating Iridotrabecular touch 
Fig. 10 Summary ROC plot for studies investigating iridotrabecular touch

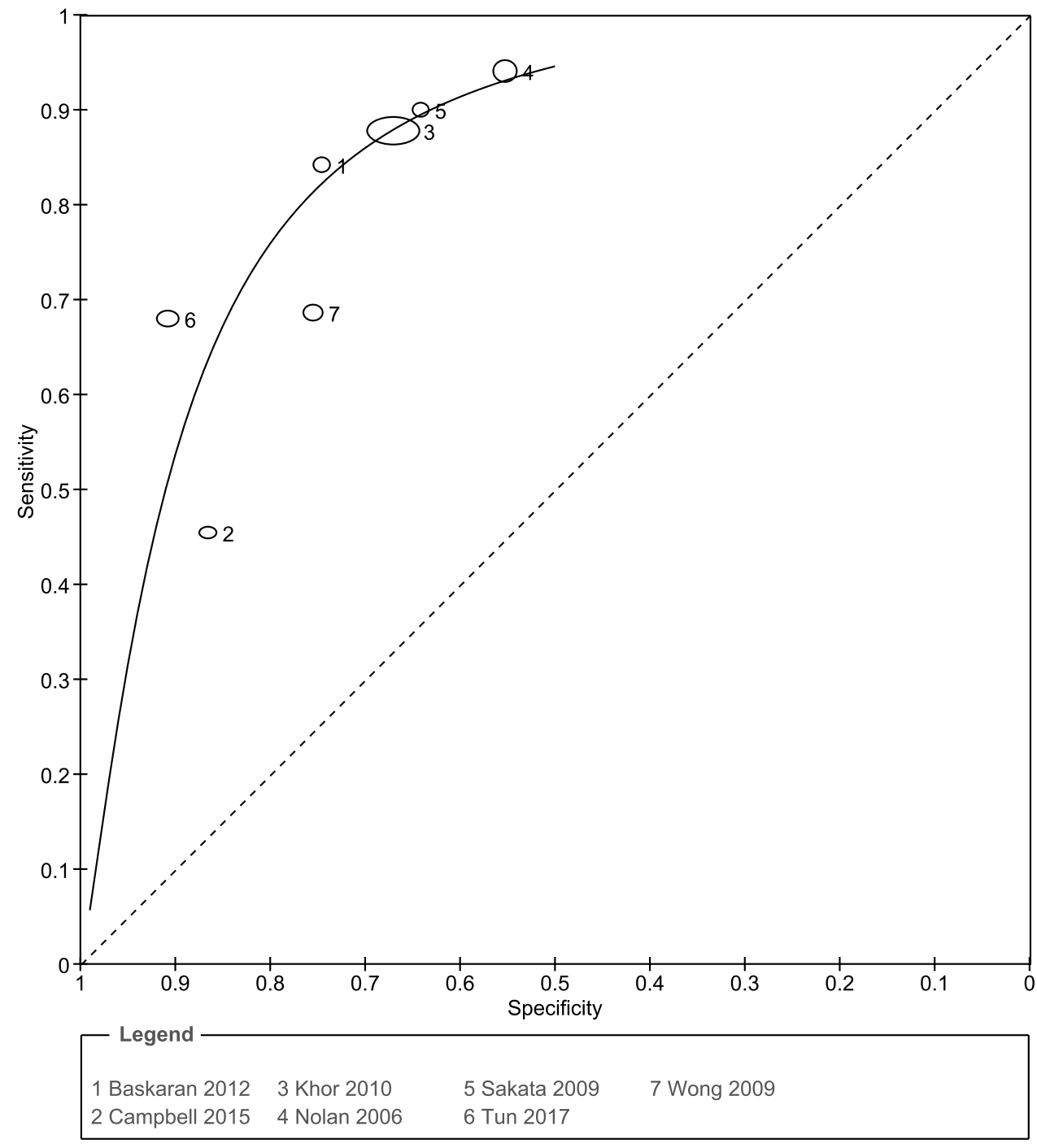

\begin{tabular}{|c|c|c|c|c|c|c|c|c|}
\hline Study & TP & FP & FN & TN & Sensitivity $(95 \% \mathrm{CI})$ & Specificity $(95 \% \mathrm{CI})$ & Sensitivity (95\% CI) & Specificity $(95 \% \mathrm{CI})$ \\
\hline Porporato 2018 & 318 & 340 & 58 & 1141 & $0.85[0.81,0.88]$ & $0.77[0.75,0.79]$ & $=$ & \\
\hline Baskaran 2013 & 12 & 28 & 1 & 99 & $0.92[0.64,1.00]$ & $0.78[0.70,0.85]$ & & \\
\hline Melese 2016 & 29 & 6 & 2 & 32 & $0.94[0.79,0.99]$ & $0.84[0.69,0.94]$ & & 1 \\
\hline
\end{tabular}

Fig. 11 Forest plot for studies investigating ITC index

not been shown on a summary ROC plot to avoid facilitating an inappropriate indirect comparison between index parameters. Li et al. (2019) found that ACV showed a sensitivity of $90.0 \%$ and specificity of $97.1 \%$ [35]. ACA was analysed in two studies and the results varied significantly $[44,38]$ with sensitivity ranging from 75.0 to $100.0 \%$ and specificity ranging from to 66.7 to $97.6 \%$. LV has been assessed in a single community based study by Tan et al. (2012) with 1465 eyes [30]. The results showed a reasonable ability to detect angle closure (sensitivity $85.7 \%$, specificity $77.5 \%$, PPV 51.0\%, NPV 95.2\%). TICV, ITC area and ITC length were analysed by Melese et al. (2016) [37]. TICV achieved $100 \%$ specificity and $87 \%$ specificity. ITC area had good results (sensitivity 93\%, specificity $87 \%$ ). The results for ITC length were more the modest. ARA has only been assessed in two studies with variable sensitivity (range 74.0-91.3) and specificity (range 69.2-100).

Nongpiur et al. (2013) assessed a stepwise logistic regression model that uses multiple parameters to determine an angle closure score and estimate a probability of angle closure [25, 26, 32]. Data for two different thresholds were presented, with one weighted toward a higher sensitivity of 
Fig. 12 Summary ROC plot for studies investigating ITC index

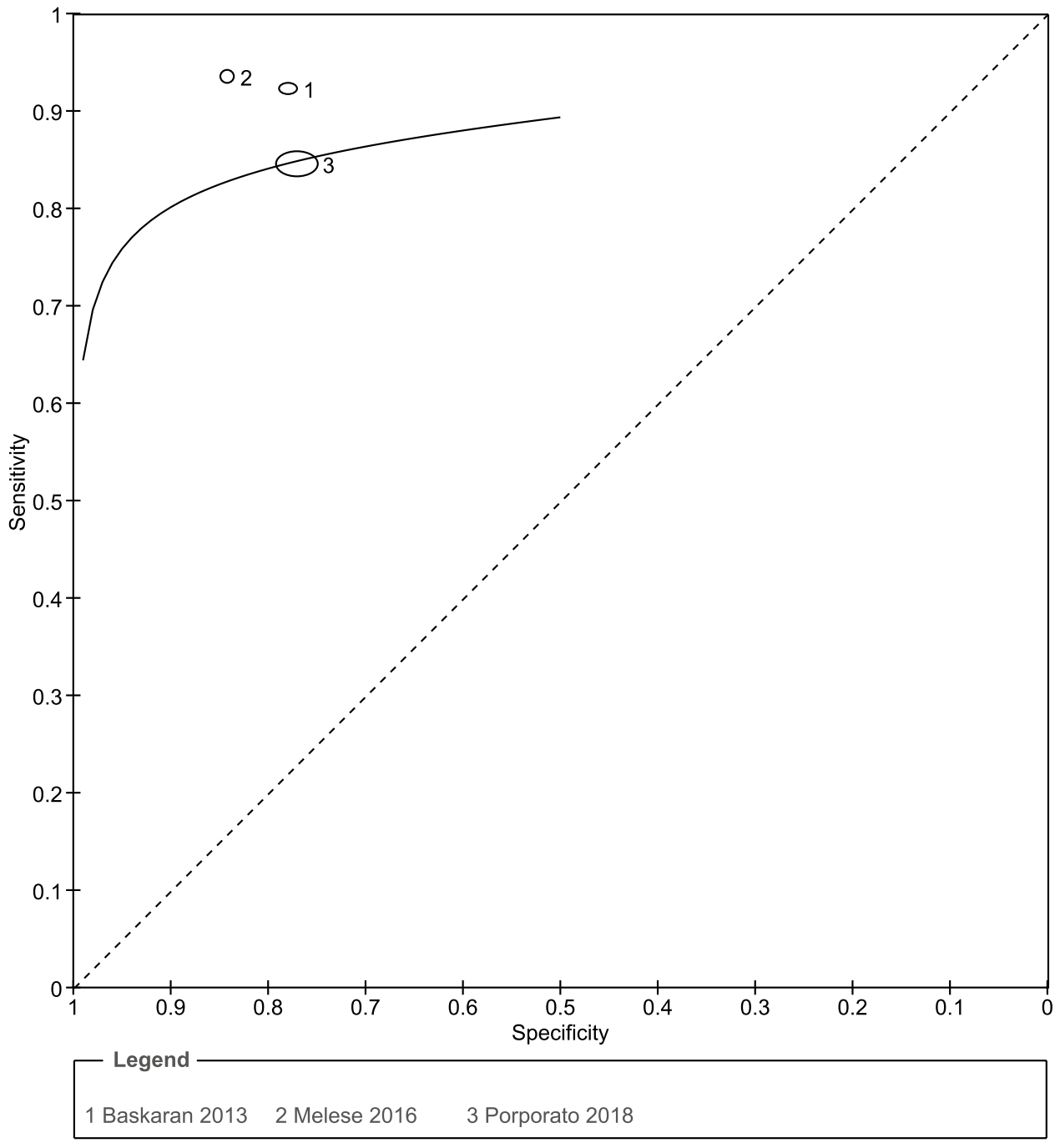

\begin{tabular}{|c|c|c|c|c|c|c|c|c|}
\hline Study & TP & FP & FN & TN & Sensitivity $(95 \% \mathrm{CI})$ & Specificity (95\% Cl) & Sensitivity $(95 \% \mathrm{Cl})$ & Specificity $(95 \% \mathrm{Cl})$ \\
\hline Dabasia 2015 & 14 & 10 & 3 & 51 & $0.82[0.57,0.96]$ & $0.84[0.72,0.92]$ & & 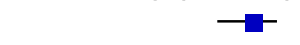 \\
\hline Hong 2009 & 36 & 1 & 5 & 31 & $0.88[0.74,0.96]$ & $0.97[0.84,1.00]$ & & \\
\hline Li 2019 & 35 & 4 & 3 & 33 & $0.92[0.79,0.98]$ & $0.89[0.75,0.97]$ & & \\
\hline
\end{tabular}

Fig. 13 Forest plot for studies investigating ACD

96\% (NPV 99\%) and the other weighted toward a higher specificity of $89 \%$ (PPV 69\%).

\section{Ability to predict development of angle closure at 4 years}

Nongpiur et al.(2017) and Baskaran et al.(2015) assessed models that use baseline AS-OCT measurements to predict angle closure by gonioscopy four years later (Fig. 16) [31, 32]. The two studies were conducted at the same community clinic in Singapore and used the exact same participants $(n=342)$. The participants were limited to those that did not have angle closure by gonioscopy at baseline. Nongpiur et al. (2017) showed the sensitivity of LV and AOD750 to be $64.6 \%$ and $95.8 \%$ respectively. Baskaran et al. (2015) assessed for the number of quadrants with presence of iridotrabecular touch. When 1 quadrant was used as the threshold, the sensitivity was $100 \%$. When 4 quadrant was used as the threshold, the specificity was above $97 \%$.

\section{Comparisons between index parameters}

In the paired studies by Narayanaswamy et al. (2010) [29] and Tan et al. (2012) [30], AOD, TISA and LV were shown 
Fig. 14 Summary ROC plot for studies investigating ACD

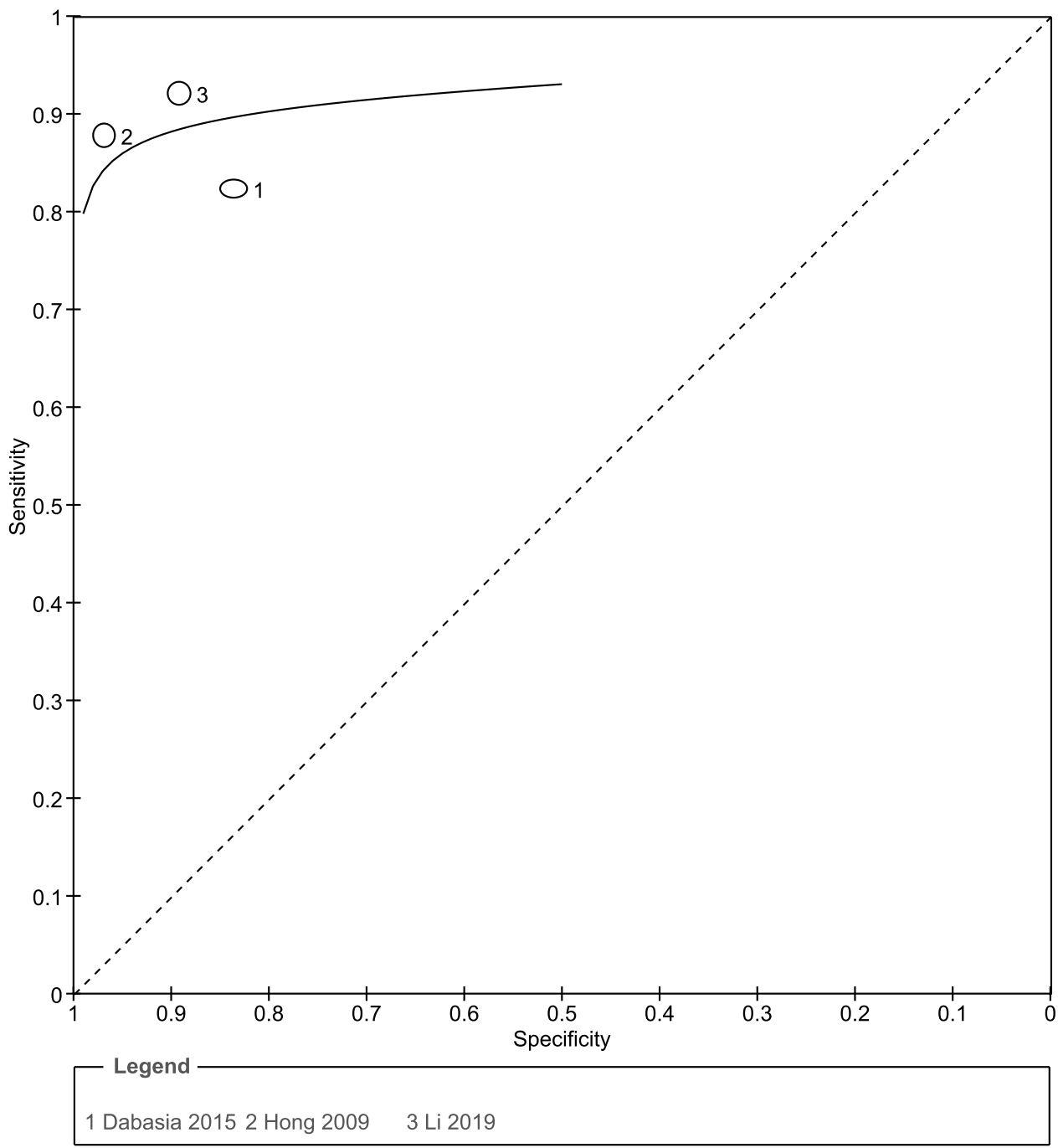

to be significantly more specific than ARA (AOD: 0.77 (95\% CI 0.75-0.80), TISA: 0.77 (95\% CI 0.74-0.79), LV: 0.77 (95\% CI 0.75-0.80), ARA: 0.69 (95\% CI 0.66-0.72)). The two longitudinal studies showed that LV was more specific than all other tested parameters, but it was less sensitive than AOD and a stepwise logistic model. However, Nongpiur et al. (2017) did not find a significant difference between parameters when comparing AUC values. These conclusions are only applicable at the specific positivity thresholds used in the studies. There is not enough information to make any further conclusions about the relative accuracy of index parameters.

\section{Discussion}

Optical coherence tomography has made an incredible impact in the field of ophthalmology. It is proven to be cost-effective and has become a key imaging modality for many eye conditions including glaucoma and neovascular age-related macular degeneration [49, 50]. Our systematic review focused on the evidence of the diagnostic accuracy of AS-OCT in detecting angle closure. We were able to produce summary ROC curves and show that AS-OCT may reach above $90 \%$ in sensitivity or specificity for detecting angle closure as defined by gonioscopy. However, the studies that showed the best results for AS-OCT were at often high risk of bias due to their study design. There is not enough evidence to conclude that any individual parameter is significantly better or worse for detecting angle closure. Similarly, there is not enough evidence to conclude that horizontal, vertical or circumferential AS-OCT scans perform differently.

AS-OCT allows excellent visualisation of the anterior angle and has several key advantages over gonioscopy. It is non-contact, more comfortable for the patient and requires little training to use. The objective measurements are ideal for ongoing monitoring of glaucoma patients and suspects. However, AS-OCT cannot replace gonioscopy which remains the reference standard as it is useful for 


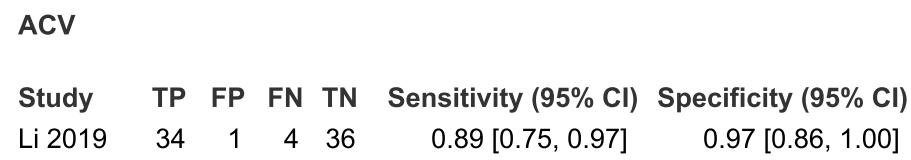

ARA
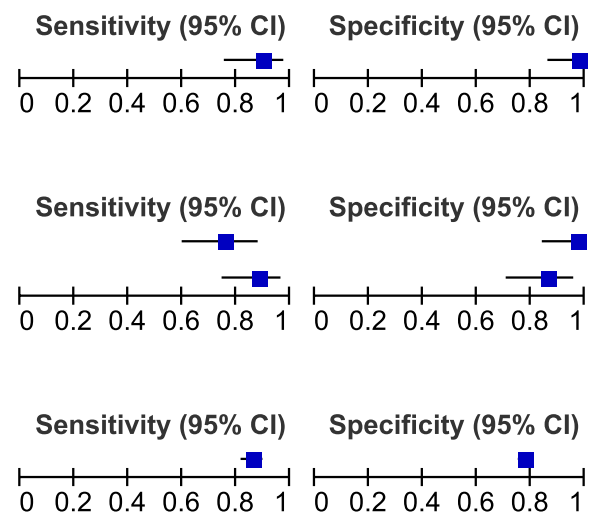
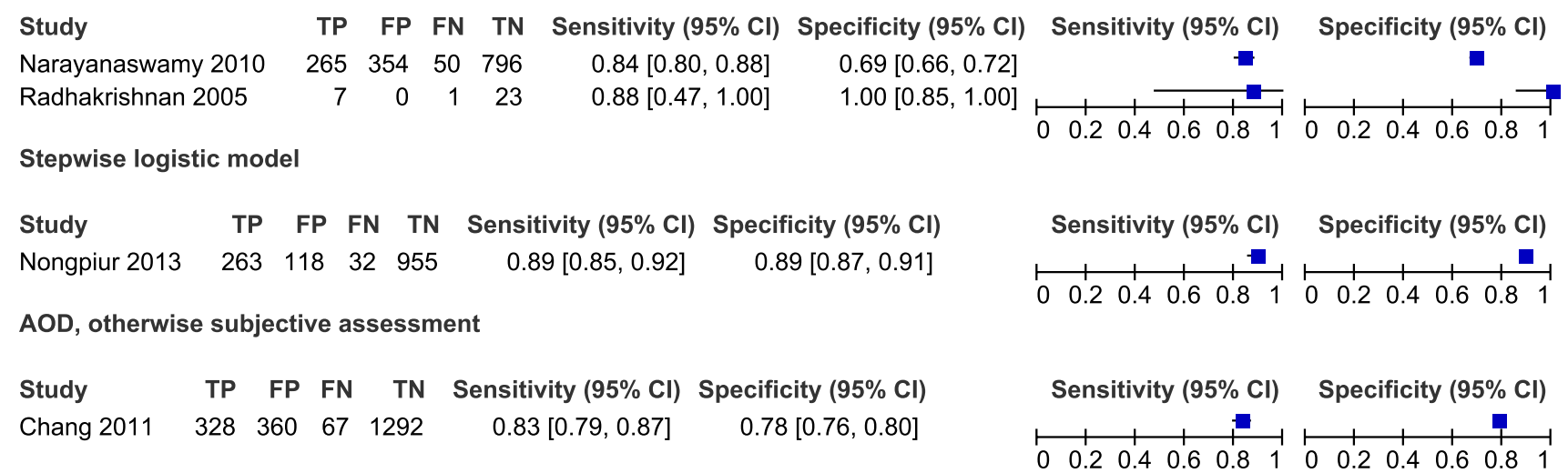

Fig. 15 Forest plot for studies investigating remaining parameters

purposes other than detecting angle closure including assessment of neovascularisation, peripheral anterior synechiae, pigment dispersion syndrome and angle recession. A dynamic assessment with indentation is also only possible with a gonioscopy lens. Furthermore, gonioscopy facilitates 360-degree visualisation of the anterior angle, whereas AS-OCT often only provides a small number of axis scans, preventing a complete assessment.

Time for data acquisition using the AS-OCT varies with different anterior angle parameters and is an important factor for clinicians from a practical perspective. AOD and TISA currently require manual input from the clinician to locate the scleral spur which may slow down the testing procedure, especially if multiple quadrants are assessed. This requirement for subjective input may also introduce error. The practicality of AOD and TISA is further reduced by frequently poor visualisation of the scleral spur. The largest study by Narayanaswamy had to exclude $25 \%$ of eyes from their analysis for this reason [29]. However, the OCT device software is improving over time and it may 
AOD-Longitudinal

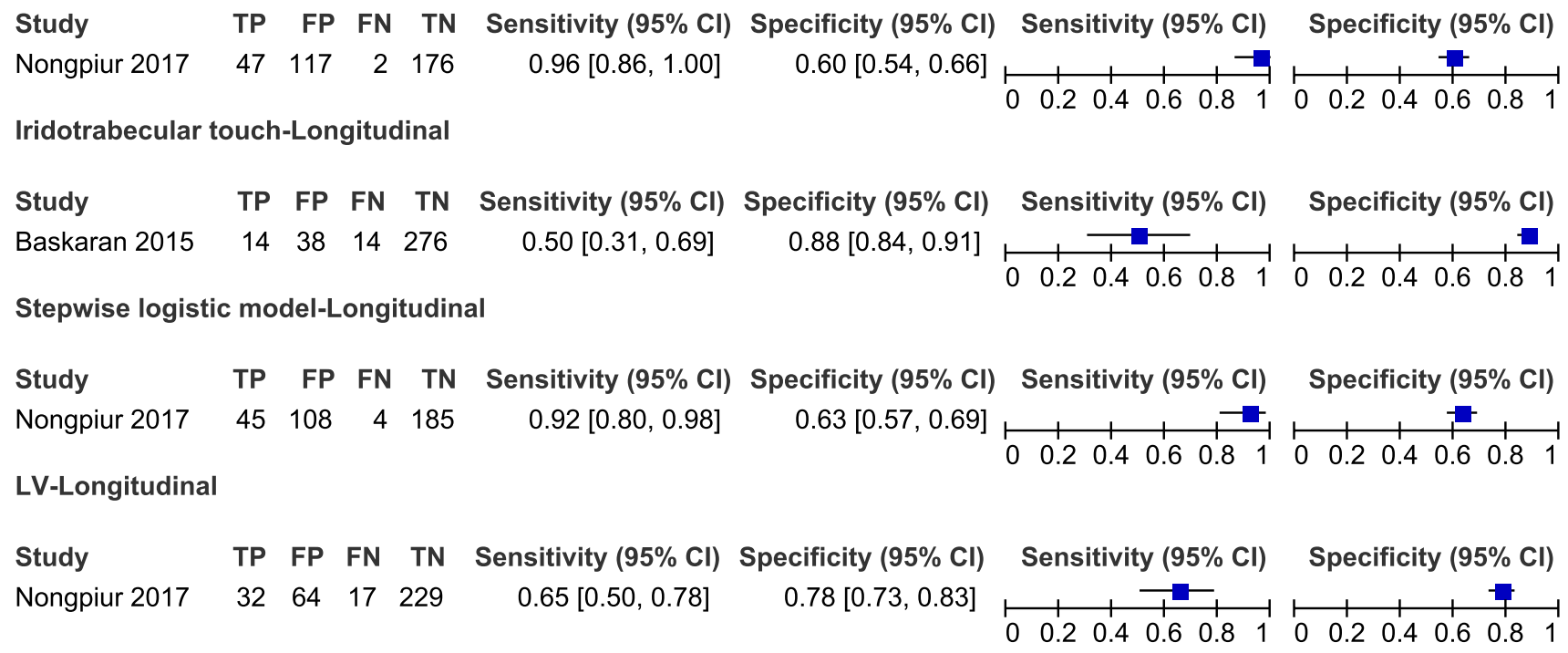

Fig. 16 Forest plot for longitudinal studies

soon be possible that results for all parameters are generated automatically.

The two included longitudinal studies showed that iridotrabecular touch and AOD750 have sensitivity above $95 \%$ for detecting eyes that would develop closed angles by gonioscopy 4 years later $[31,32]$. However, they reported a high false-positive rate, leading to poor specificity. The longitudinal design allows us to analyse the natural history of eyes that are classified to have closed angles by AS-OCT but open by gonioscopy. These eyes correspond to false-positive data points in other studies. The results suggest that the eyes determined to have angle closure by AS-OCT should be carefully monitored as they are at higher risk of developing angle closure on gonioscopy.

Our review gives insight into possible clinical applications for AS-OCT in detecting angle closure. The good sensitivity corresponds to a strong NPV (reaching above 95\%) and suggests that there might be a place for AS-OCT in ruling out angle closure. A negative result could be very reassuring in situations where a practitioner skilled in gonioscopy is not available. Potential use cases include telehealth consultations or in primary care practice. These situations are particularly relevant in the context of the COVID-19 pandemic when this review was written.

When the condition being tested for has a low prevalence in the population being tested, it is ideal if the specificity is high otherwise the PPV will be low. Despite AS-OCT generally showing specificity above $80 \%$, the prevalence of angle closure is low enough that the PPV was often less than $50 \%$. A positive result becomes difficult to interpret and would necessitate further testing.
We do not currently know if these false-positive errors are truly errors or if they represent eyes at greater risk of angle closure and glaucomatous damage. This uncertainty is partially caused by using gonioscopy to define angle closure. Nolan et al. (2006) have argued that gonioscopy may not be the most appropriate reference standard as it may miss cases of angle closure [47]. That if gonioscopy was being tested against AS-OCT as the reference standard, it would be shown to have low sensitivity and high specificity.

Another possible interpretation of the false-positive errors is that they represent pre-gonioscopic angle closure; that the eyes only determined to have angle closure by AS-OCT are at higher risk of glaucoma but have not yet progressed to angle closure by gonioscopy. Specifically, AS-OCT could allow for early detection of angle closure. This is supported by the two longitudinal studies that showed AS-OCT was very sensitive in detecting eyes that would progress from open to closed on gonioscopy $[31,32]$. More longitudinal studies are needed to better determine the long-term risk of glaucoma and angle closure for patients that are deemed to be closed on AS-OCT but open by gonioscopy.

Due to its strong NPV, ease of use, objective measurements and possibility for earlier detection, AS-OCT may be ideal for initial assessment of patients at higher risk of angle closure. This may include patients from higher risk populations such as Asians, or those with structural risk factors. As an added benefit, AS-OCT gives information on iris configuration and anterior eye structure. This enables assessment of structural risk factors including pupillary block, large lens vault and large iris area [51-53]. 
When assessing the utility of AS-OCT for detecting angle closure, we should consider the shortcomings of our current tools. Many eyes with angle closure disease go unrecognised in developed countries and the rates are even worse in developing Asian countries. There are not enough clinicians skilled in gonioscopy to adequately assess for angle closure on a large scale. In this context, AS-OCT provides a tool for screening where there is very little else available. This provides an avenue to detect currently undiagnosed angle closure and reduce the long-term burden of blindness. Unfortunately, developing countries are currently unlikely to have access to an OCT device due to the prohibitive cost. However, this may change as the cost of technology decreases with time. Clinicians should consider the strengths and limitations of AS-OCT to determine how it may be safely used for their specific clinical needs.

Our review has been the first to perform a meta-analysis of data that assesses the accuracy of AS-OCT for detecting angle closure against gonioscopy as a reference standard. A review by the American Academy of Ophthalmology (2013) identified that AS-OCT may provide supplemental information when used alongside gonioscopy [13], whereas we found that AS-OCT has a strong NPV and may have a place in screening for angle closure. We found that AS-OCT may have specificity above $80 \%$ when certain parameters and thresholds are used. This contrasts with a review by Porporato et al. (2018) that reported the specificity to be low [14]. Despite this, we agreed that the PPV was often poor. It remains to be seen how the clinical utility of AS-OCT compares against other anterior chamber analysis modalities including Scheimpflug photography and Scanning Peripheral Anterior Chamber Depth Analyser.

There were several limitations of our review. There was substantial variation between studies in areas including gonioscopy criteria, AS-OCT positivity threshold and OCT device being used. We were therefore limited in what statistical analyses we were able to perform, making it difficult to draw firm conclusions on the utility of AS-OCT. We were unable to determine summary sensitivity and specificity values for each index parameter. We were also unable to perform a quantitative analysis of heterogeneity or quantitative comparison between index parameters.

To reduce heterogeneity, future studies should consider using non-visibility of 2 quadrants of PTM as the gonioscopy criterion if it matches clinical practice. Another approach would be to include results for several gonioscopy criteria as some studies have done. Future studies should analyse data using positivity thresholds established in previous studies if possible. If a new threshold is established, they should also validate their results using a separate population or by using a cross-validation procedure. Future studies should also avoid a case-control design.

\section{Conclusion}

Although the current literature is heterogenous in methodology, AS-OCT demonstrates good sensitivity and NPV. There are not enough clinicians skilled in gonioscopy to adequately assess for angle closure on a large scale, particularly in developing Asian countries. In this context, AS-OCT allows for earlier detection and provides a tool for screening where there is very little else available. This provides an avenue to detect currently undiagnosed angle closure and reduce the long-term burden of blindness. However, AS-OCT is not yet able to replace gonioscopy. Clinicians should consider whether the diagnostic accuracy of AS-OCT is acceptable for their specific clinical use before adopting it. More studies are needed to determine the utility of AS-OCT, including longitudinal studies to determine the significance of eyes classified to have closed angles by AS-OCT but open on gonioscopy.

\section{Appendix 1}

Full search strategy for Medline database.o

1. gonio*.mp. or exp Gonioscopy/

2. exp Tomography, Optical Coherence/ or optical coherence tomography.mp.

3. OCT.mp.

4. 2 or 3

5. narrow angle.mp.

6. closed angle.mp.

7. angle closure.mp.

8. Glaucoma, Angle-Closure/

9. 5 or 6 or 7 or 8

10. detect*.mp.

11. diagnos*.mp.

12. identif*.mp.

13. screen*.mp.

14. 10 or 11 or 12 or 13

15. 1 and 4 and 9 and 14

Supplementary Information The online version contains supplementary material available at https://doi.org/10.1007/s00417-021-05271-4. 
Acknowledgements Many thanks to Dr. Daisy Shu of Schepens Eye Research Institute, Harvard Medical School for her time, expertise and advise in writing this paper.

\section{Declarations}

Ethics approval The need for ethics approval was waived by the institutional review board as this is a systematic review of existing literature.

Consent to participate Not applicable.

Consent for publication Not applicable.

Competing interests The authors declare no competing interest.

\section{References}

1. Tham Y-C, Li X, Wong TY et al (2014) Global prevalence of glaucoma and projections of glaucoma burden through 2040: a systematic review and meta-analysis. Ophthalmology 121:20812090. https://doi.org/10.1016/j.ophtha.2014.05.013

2. Quigley HA, Broman AT (2006) The number of people with glaucoma worldwide in 2010 and 2020. Br J Ophthalmol 90:262-267. https://doi.org/10.1136/bjo.2005.081224

3. Asian Pacific Glaucoma Society (APGS) (2016) Asia Pacific Glaucoma Guidelines, 3rd edn. Amsterdam: Kugler Publications

4. Friedman DS, Foster PJ, Aung T, He M (2012) Angle closure and angle-closure glaucoma: what we are doing now and what we will be doing in the future. Clin Experiment Ophthalmol 40:381-387. https://doi.org/10.1111/j.1442-9071.2012. 02774.X

5. Liang Y, Friedman DS, Zhou Q et al (2011) Prevalence and characteristics of primary angle-closure diseases in a rural adult Chinese population: the Handan eye study. Invest Ophthalmol Vis Sci 52:8672-8679. https://doi.org/10.1167/iovs.11-7480

6. Senthil S, Garudadri C, Khanna RC, Sannapaneni K (2010) Angle Closure in the Andhra Pradesh Eye Disease Study. Ophthalmology 117:1729-1735. https://doi.org/10.1016/j.ophtha.2010.01.021

7. Thomas R, Parikh R, Muliyil J, Kumar RS (2003) Five-year risk of progression of primary angle closure to primary angle closure glaucoma: a population-based study. Acta Ophthalmol Scand 81:480-485. https://doi.org/10.1034/j.1600-0420.2003.00135.x

8. Pandit RJ, Taylor R (2000) Mydriasis and glaucoma: exploding the myth A systematic review. Diabet Med 17:693-699. https:// doi.org/10.1046/j.1464-5491.2000.00368.x

9. Parikh R, Mathai A, Parikh S et al (2008) Understanding and using sensitivity, specificity and predictive values. Indian J Ophthalmol 56:45-50

10. Campbell P, Agarwal R, Redmond T et al (2014) Repeatability and comparison of anterior chamber angle assessment tests. Invest Ophthalmol Vis Sci 55:933

11. Lee CS, Lee ML, Yanagihara RT, Lee AY (2020) Predictors of narrow angle detection rate-a longitudinal study of Massachusetts residents over 1.7 million person years. Eye 1-7. https://doi. org/10.1038/s41433-020-1003-0

12. Asia-Pacific Glaucoma Society (2016) Asia Pacific Glaucoma Guidelines, 3rd edn. Amsterdam: Kugler Publications

13. Smith SD, Singh K, Lin SC et al (2013) Evaluation of the anterior chamber angle in glaucoma: a report by the american academy of ophthalmology. Ophthalmology 120:1985-1997. https://doi.org/ 10.1016/j.ophtha.2013.05.034

14. Porporato N, Baskaran M, Aung T (2018) Role of anterior segment optical coherence tomography in angle-closure disease: a review. Clin Experiment Ophthalmol 46:147-157. https://doi.org/ 10.1111/ceo.13120

15. Chansangpetch S, Rojanapongpun P, Lin SC (2018) Anterior segment imaging for angle closure. Am J Ophthalmol 188:xvi-xxix. https://doi.org/10.1016/j.ajo.2018.01.006

16. Jindal A, Ctori I, Virgili G et al (2020) Non-contact tests for identifying people at risk of primary angle closure glaucoma. Cochrane Database Syst Rev. https://doi.org/10.1002/14651858. CD012947.pub2

17. Whiting PF, Rutjes AWS, Westwood ME et al (2011) QUADAS-2: a revised tool for the quality assessment of diagnostic accuracy studies. Ann Intern Med 155:529-536. https://doi.org/10.7326/ 0003-4819-155-8-201110180-00009

18. The Cochrane Collaboration (2020) Review Manager (RevMan), Computer software, Version 5.4. http://revman.cochrane.org. Accessed 17 Aug 2020

19. Macaskill P, Gatsonis C, Deeks JJ, et al (2010) Chapter 10: analysing and presenting results. In: Cochrane Handbook for Systematic Reviews of Diagnostic Test Accuracy Version 1.0. The Cochrane Collaboration. http://srdta.cochrane.org. Accessed 17 Aug 2020

20. Takwoingi Y, Deeks J (2010) MetaDAS: a SAS macro for metaanalysis of diagnostic accuracy studies, Computer software, Version 1.3.0. http://srdta.cochrane.org. Accessed 17 Aug 2020

21. SAS Institute Inc (2016) SAS Software, Version 9.4 of the SAS System for Windows, Computer software. https://www.sas.com. Accessed 17 Aug 2020

22. Youden WJ (1950) Index for rating diagnostic tests. Cancer 3:32-35. https://doi.org/10.1002/1097-0142(1950)3:1\%3c32:: AID-CNCR2820030106\%3e3.0.CO;2-3

23. Takwoingi Y, Leeflang MM, Deeks JJ (2013) Empirical evidence of the importance of comparative studies of diagnostic test accuracy. Ann Intern Med 158:544-554

24. Park SB, Sung KR, Kang SY et al (2011) Assessment of narrow angles by gonioscopy, Van Herick method and anterior segment optical coherence tomography. Jpn J Ophthalmol 55:343-350. https://doi.org/10.1007/s10384-011-0036-0

25. Nongpiur ME, Haaland BA, Friedman DS et al (2012) Classification algorithms based on anterior segment optical coherence tomography measurements for detection of angle closure. Ophthalmology 120:48-54. https://doi.org/10.1016/j.ophtha.2012.07. 005

26. Nongpiur ME, Haaland BA, Perera SA et al (2013) Development of a score and probability estimate for detecting angle closure based on anterior segment optical coherence tomography. Am J Ophthalmol 157:32-38.e1. https://doi.org/10.1016/j.ajo.2013.09. 012

27. Chang DS, Sakata LM, Aung T et al (2011) Single versus sequential testing with scanning peripheral anterior chamber depth analyser, IOLMaster and anterior segment optical coherence tomography for the detection of narrow angles. Br J Ophthalmol 95:1410-1414. https://doi.org/10.1136/bjo.2009.175869

28. Khor W-B, Sakata LM, Friedman DS et al (2010) Evaluation of scanning protocols for imaging the anterior chamber angle with anterior segment-optical coherence tomography. J Glaucoma 19:365-368. https://doi.org/10.1097/IJG.0b013e3181bf4dd5

29. Narayanaswamy A, Sakata LM, He M-G et al (2010) Diagnostic performance of anterior chamber angle measurements for detecting eyes with narrow angles: an anterior segment OCT study. Arch Ophthalmol Chic Ill 128:1321-1327. https://doi.org/10.1001/ archophthalmol.2010.231

30. Tan GS, He M, Zhao W et al (2012) Determinants of lens vault and association with narrow angles in patients from Singapore. 
Am J Ophthalmol 154:39-46. https://doi.org/10.1016/j.ajo.2012. 01.015

31. Baskaran M, Iyer JV, Narayanaswamy AK et al (2015) Anterior Segment Imaging Predicts Incident Gonioscopic Angle Closure. Ophthalmology 122:2380-2384. https://doi.org/10.1016/j.ophtha. 2015.07.030

32. Nongpiur ME, Aboobakar IF, Baskaran M et al (2017) Association of baseline anterior segment parameters with the development of incident gonioscopic angle closure. JAMA Ophthalmol 135:252-258. https://doi.org/10.1001/jamaophthalmol.2016.5847

33. Porporato N, Baskaran M, Tun TA et al (2018) Assessment of circumferential angle closure with swept-source optical coherence tomography: a community based study. Am J Ophthalmol 199:133-139. https://doi.org/10.1016/j.ajo.2018.11.015

34. Porporato N, Baskaran M, Tun TA, et al (2019) Understanding diagnostic disagreement in angle closure assessment between anterior segment optical coherence tomography and gonioscopy. Br J Ophthalmol. https://doi.org/10.1136/bjophthalm ol-2019-314672

35. Li F, Zhou R, Gao K et al (2019) Volumetric parameters-based differentiation of narrow angle from open angle and classification of angle configurations: an SS-OCT study. Br J Ophthalmol 104:92-97. https://doi.org/10.1136/bjophthalmol-2018-313386

36. Tun TA, Baskaran M, Tan SS et al (2017) Evaluation of the anterior segment angle-to-angle scan of cirrus high-definition optical coherence tomography and comparison with gonioscopy and with the visante OCT. Invest Ophthalmol Vis Sci 58:59-64. https://doi. org/10.1167/iovs.16-20886

37. Melese EK, Chan JD, Blieden LS et al (2016) Determination and validation of thresholds of anterior chamber parameters by dedicated anterior segment optical coherence tomography. Am J Ophthalmol 169:208-217. https://doi.org/10.1016/j.ajo.2016.06. 032

38. Dabasia PL, Edgar DF, Murdoch IE, Lawrenson JG (2015) Noncontact screening methods for the detection of narrow anterior chamber angles. Invest Ophthalmol Vis Sci 56:3929-3935. https:// doi.org/10.1167/iovs.15-16727

39. Campbell P, Redmond T, Agarwal R et al (2015) Repeatability and comparison of clinical techniques for anterior chamber angle assessment. Ophthalmic Physiol Opt J Br Coll Ophthalmic Opt Optom 35:170-178. https://doi.org/10.1111/opo.12200

40. Qin B, Francis BA, Li Y et al (2013) Anterior chamber angle measurements using Schwalbe's line with high-resolution fourierdomain optical coherence tomography. J Glaucoma 22:684-688. https://doi.org/10.1097/IJG.0b013e318264b921

41. Baskaran M, Ho S-W, Tun TA et al (2013) Assessment of circumferential angle-closure by the iris-trabecular contact index with swept-source optical coherence tomography. Ophthalmology 120:2226-2231. https://doi.org/10.1016/j.ophtha.2013.04.020

42. Baskaran M, Aung T, Friedman DS et al (2012) Comparison of EyeCam and anterior segment optical coherence tomography in detecting angle closure. Acta Ophthalmol (Copenh) 90:e621e625. https://doi.org/10.1111/j.1755-3768.2012.02510.x
43. Grewal DS, Brar GS, Jain R, Grewal SPS (2011) Comparison of Scheimpflug imaging and spectral domain anterior segment optical coherence tomography for detection of narrow anterior chamber angles. Eye Lond Engl 25:603-611

44. Hong S, Yi J-H, Kang SY et al (2009) Detection of occludable angles with the pentacam and the anterior segment optical coherence tomography. Yonsei Med J 50:525-528

45. Sakata LM, Wong TTL, Wong H-T et al (2009) Comparison of Visante and slit-lamp anterior segment optical coherence tomography in imaging the anterior chamber angle. Eye Lond Engl 24:578-587. https://doi.org/10.1038/eye.2009.141

46. Wong H-T, Chua JLL, Sakata LM et al (2009) Comparison of slitlamp optical coherence tomography and scanning peripheral anterior chamber depth analyzer to evaluate angle closure in Asian eyes. Arch Ophthalmol Chic Ill 127:599-603. https://doi.org/10. 1001/archophthalmol.2009.41

47. Nolan WP, See JL, Chew PTK et al (2006) Detection of primary angle closure using anterior segment optical coherence tomography in Asian eyes. Ophthalmology 114:33-39. https://doi.org/10. 1016/j.ophtha.2006.05.073

48. Radhakrishnan S, Goldsmith J, Huang D et al (2005) Comparison of optical coherence tomography and ultrasound biomicroscopy for detection of narrow anterior chamber angles. Arch Ophthalmol 123:1053-1059. https://doi.org/10.1001/archopht.123.8.1053

49. Fujimoto J, Swanson E (2016) The development, commercialization, and impact of optical coherence tomography. Invest Ophthalmol Vis Sci 57:OCT1-OCT13. https://doi.org/10.1167/iovs. 16-19963

50. Windsor MA, Sun SJJ, Frick KD et al (2018) Estimating public and patient savings from basic research-a study of optical coherence tomography in managing antiangiogenic therapy. Am J Ophthalmol 185:115-122. https://doi.org/10.1016/j.ajo.2017.09. 027

51. Nongpiur ME, Atalay E, Gong T et al (2017) Anterior segment imaging-based subdivision of subjects with primary angle-closure glaucoma. Eye Lond Engl 31:572-577. https://doi.org/10.1038/ eye.2016.267

52. Kwon J, Sung KR, Han S (2018) Long-term changes in anterior segment characteristics of eyes with different primary angle-closure mechanisms. Am J Ophthalmol 191:54-63. https://doi.org/ 10.1016/j.ajo.2018.04.005

53. Nongpiur ME, Tun TA, Aung T (2020) Anterior segment optical coherence tomography: is there a clinical role in the management of primary angle closure disease? J Glaucoma 29:60-66. https:// doi.org/10.1097/IJG.0000000000001355

Publisher's Note Springer Nature remains neutral with regard to jurisdictional claims in published maps and institutional affiliations. 